\title{
Chemical Constituents and Biological Activities of Cassia Genus: Review
}

\author{
Safaa A. Hafez ${ }^{\text {a }}$, Samir M. Osman ${ }^{\text {a }}$, Haitham A. Ibrahim ${ }^{\text {b }}$, Ahmed A. Seada ${ }^{\mathrm{c}}$, Nahla A. Ayoub ${ }^{\text {d,e }}$ \\ ${ }^{a}$ Department of Pharmacognosy, Faculty of Pharmacy, October 6 University, Giza, Egypt \\ ${ }^{\mathrm{b}}$ Department of Pharmacognosy, Faculty of Pharmacy, Helwan University, Cairo, Egypt \\ ${ }^{\mathrm{c}}$ Department of Pharmacognosy, Faculty of Pharmacy, Cairo University, Giza, Egypt \\ ${ }^{\mathrm{d}}$ Department of Pharmacognosy, Faculty of Pharmacy, Ain Shams University, Cairo, Egypt \\ ${ }^{\mathrm{e}}$ Department of Pharmacology and Toxicology, Faculty of Medicine, Umm Al Qura University, Saudi Arabia
}

\begin{abstract}
A review of chemical constituents and pharmacological activities of genus Cassia, Family Leguminosae has been presented. There are about 600 species of this genus distributed all around the world. Many of these species are still not investigated. Hence, an attempt is made to present a review on the phytochemical and biological studies of Cassia species that remain a potential source for new natural pharmacologically active components.
\end{abstract}

Keywords: Cassia genus; chemical constituents; biological activities

*Correspondence | Nahla Ayoub; Department of Pharmacognosy, Faculty of Pharmacy, Ain Shams University, Cairo, Egypt and Faculty of Medicine, UQU, KSA. Email: n.a.m.ayoub@gmail.com, naayoub@uqu.edu.sa

Citation | Hafez SA, Osman SM, Ibrahim HA, Seada AA, Ayoub NA, 2019. Chemical Constituents and Biological Activities of Cassia Genus:

Review. Arch Pharm Sci ASU 3(2): 195-227

DOI: $10.21608 / \mathrm{aps} .2019 .15746 .1008$

Print ISSN: 2356-8380. Online ISSN: 2356-8399.

Received 23 September 2019. Accepted 18 November 2019.

Copyright: ${ }^{\circledR} 2019 \mathrm{Hafez}$ et al. This is an open-access article licensed under a Creative Commons Attribution 4.0 International License (CC BY 4.0), which permits unrestricted use, distribution, and reproduction in any medium, provided the original author(s) and source are credited.

Published by: Ain Shams University, Faculty of Pharmacy

\section{INTRODUCTION}

Since ancient times, several societies have resorted to nature, mainly to plants as medical and healthy sources. Today, a great percentage of the world population, particularly in developing countries, use plants for facing the primary needs of medical assistance [1].

\section{Taxonomy}

Family Leguminosae is one of the largest families of the flowering plants. It comprises about 650 genera and 18000 species [2]. The Fabaceae or Leguminosae, commonly known as the legume, pea or bean family, is a large and economically important family of flowering plants. Plants of this family are found throughout the world, growing in many different environments and climates. The plants range in habit from giant trees to small annual herbs, with the majority being herbaceous perennials. The plants have indeterminate inflorescences, which are sometimes reduced to a single flower. The flowers have a short hypanthium and a single carpel with a short gynophore, and after fertilization produces fruits that are legumes. The leaves are usually alternate compounds and are even - or odd-pinnately compound. The name "Cassia" means "Cinnamon-like bark". Besides, 
the genus Cassia was for long ill-delimited with regards to the related Cassiinae - especially Senna (which has many medicinal important species) [3].

Leguminosae is divided into three subfamilies: Caesalpinioideae, Papilionoideae, and Mimosoideae. These sub-families are now treated as independent families due to their numerous species and named, Caesalpiniaceae, Papilionaceae, and Mimosaceae [4]. The name Caesalpinioideae is derived from the generic name Caesalpinia. The Caesalpinioideae are mainly trees distributed in the moist tropical area. Caesalpinacae represents approximately $11 \%$ of the known legume flora, with 152 genera and 2800 species [5]. Cassia is a major genus of the Caesalpiniaceae, comprising about 600 species [6].

\section{Traditional uses of Genus Cassia}

Some of them are used in traditional folk medicines as a laxative, purgative, antimalarial, ulcer healing, antidiabetic, hepatoprotective, nephroprotective, antitumor and also used in the treatment of skin infection and periodic fever throughout the tropical and subtropical region.

\section{Chemical constituents of genus Cassia}

Deep reviewing of literature concerning genus Cassia revealed the isolation and separation of different following classes of compounds: A number of authors isolated and identified several compounds from different Cassia species such as anthraquinones, anthracenes, polyphenols, fatty acids, sterols, polysaccharides and some other miscellaneous compounds from different Cassia species [7] summarized in table 1, 2, 3 and their chemical structure in Fig. 1 (Fig. 1.1-Fig. 1.21), Fig. 2 (Fig. 2.1-Fig. 2.5) and Fig. 3 (Fig. 3.1-
Fig. 3.8).

\section{Reported biological activities of species belonging to genus Cassia}

Previous evaluation of the pharmacological benefits of many Cassia species showed that most of them possess many biological activities of great importance made some of them be included in the pharmacopeia like $C$. glauca $C$. angustifolia and $C$. acutifolia. Some of these important activities such as antioxidant activity, antidiabetic, hepatoprotective activity and cytotoxic activity; all are summarized in table 4. The phytochemical constituents and biological activities of compounds isolated and identified from Cassia were searched through SciFinder that retrieves information in databases produced by Chemical Abstracts Service (CAS) as well as the MEDLINE database of the National Library of Medicine. The data was updated in March 2019, regarding chemical constituents or biological activities and Cassia keywords.

\section{CONCLUSION}

The Cassia species have been carefully studied for their phytoconstituents as well as for their biological activities. From collected data it was observed that many phytochemical constituents were isolated from different Cassia species; anthraquinones, anthracene and phenolic acids. Many biological activities were reported about Cassia species such as; antidiabetic, antioxidant hepatoprotective, cytotoxic and hypolipidemic effects. Emodin, chrysophanol, and rhein are widely distributed throughout this genus which recommends that these compounds may be chemotaxonomic markers of the genus Cassia 
Table 1. Reported anthraquinones, anthracenes and their derivatives in different Cassia species

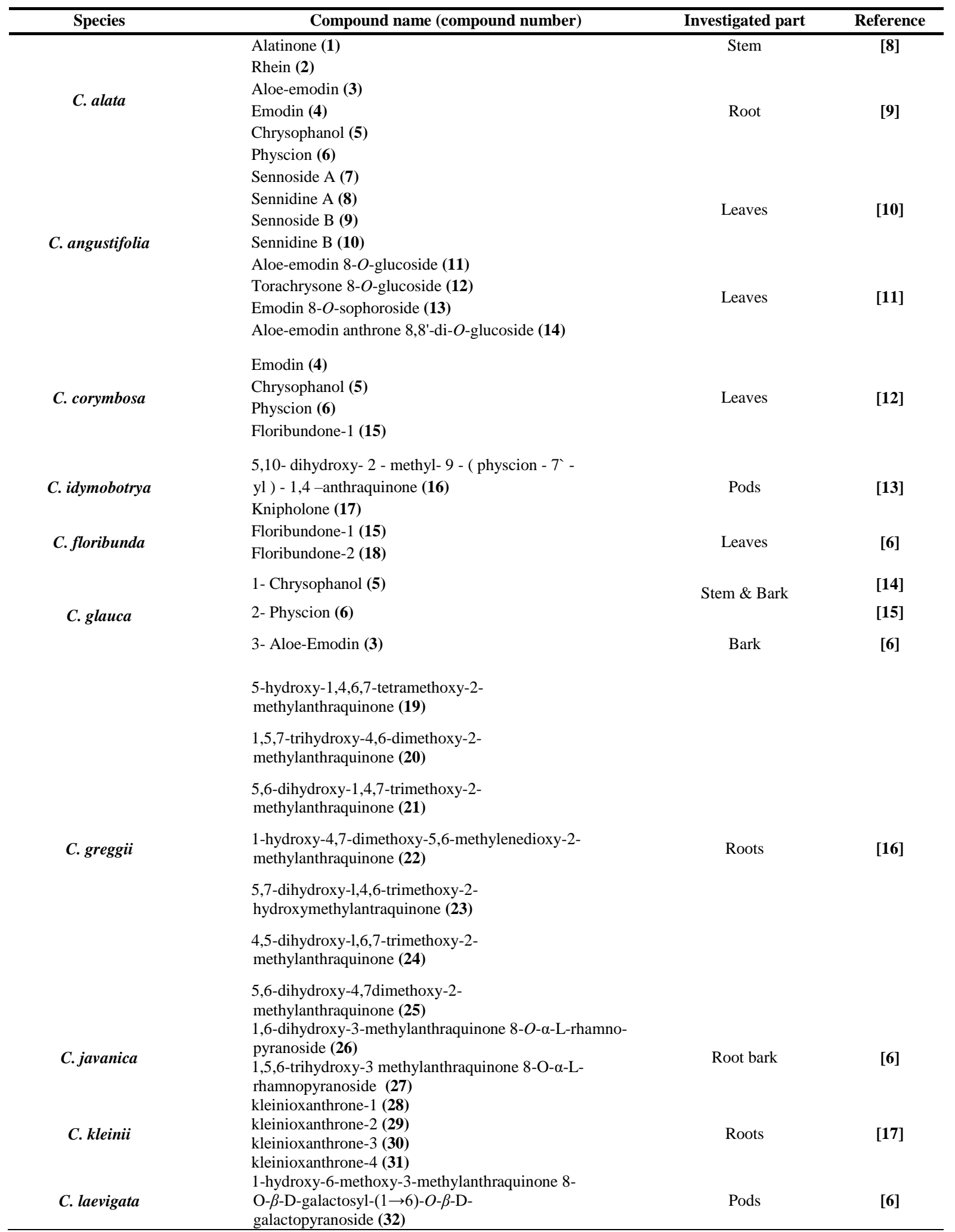




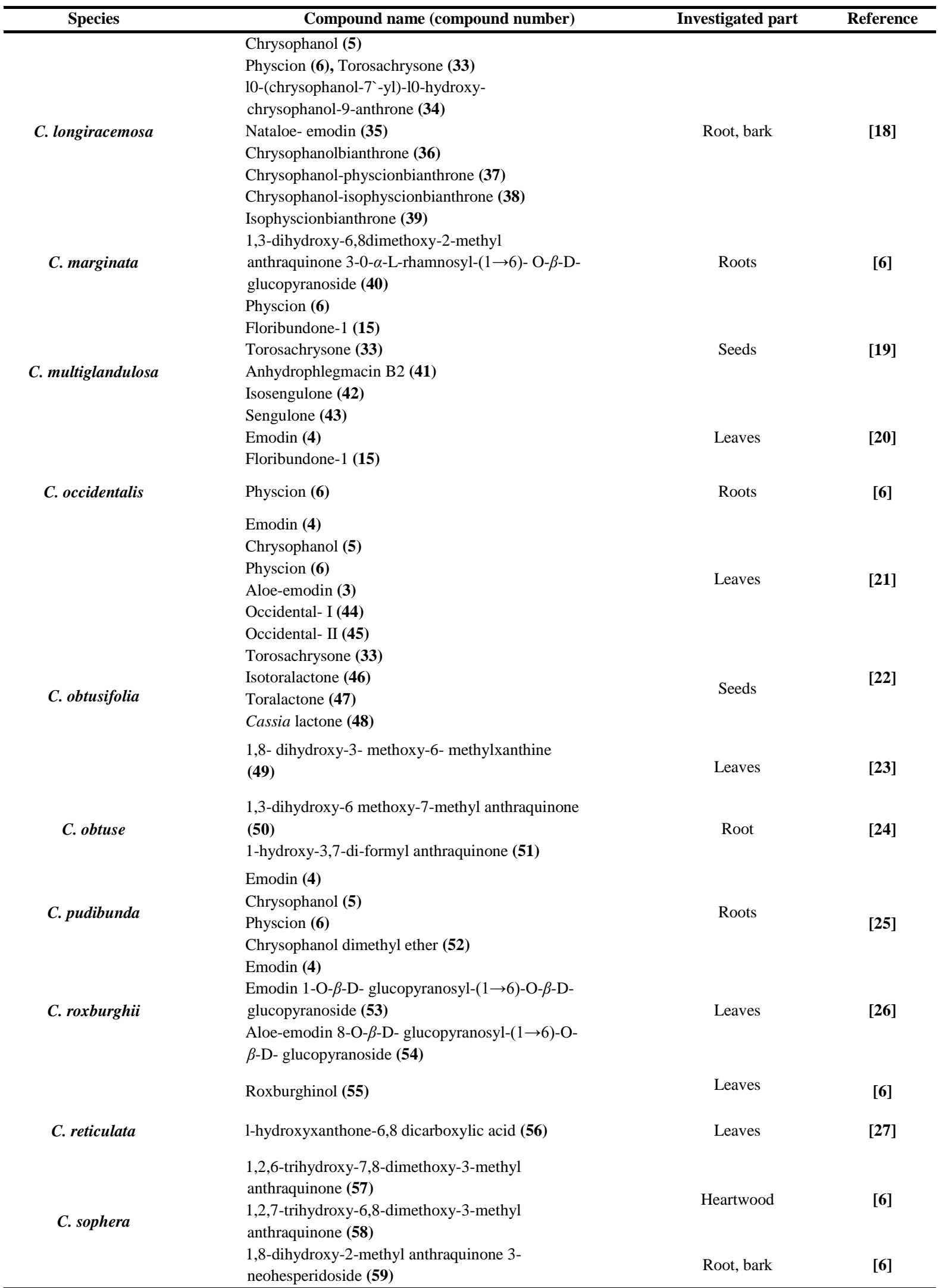




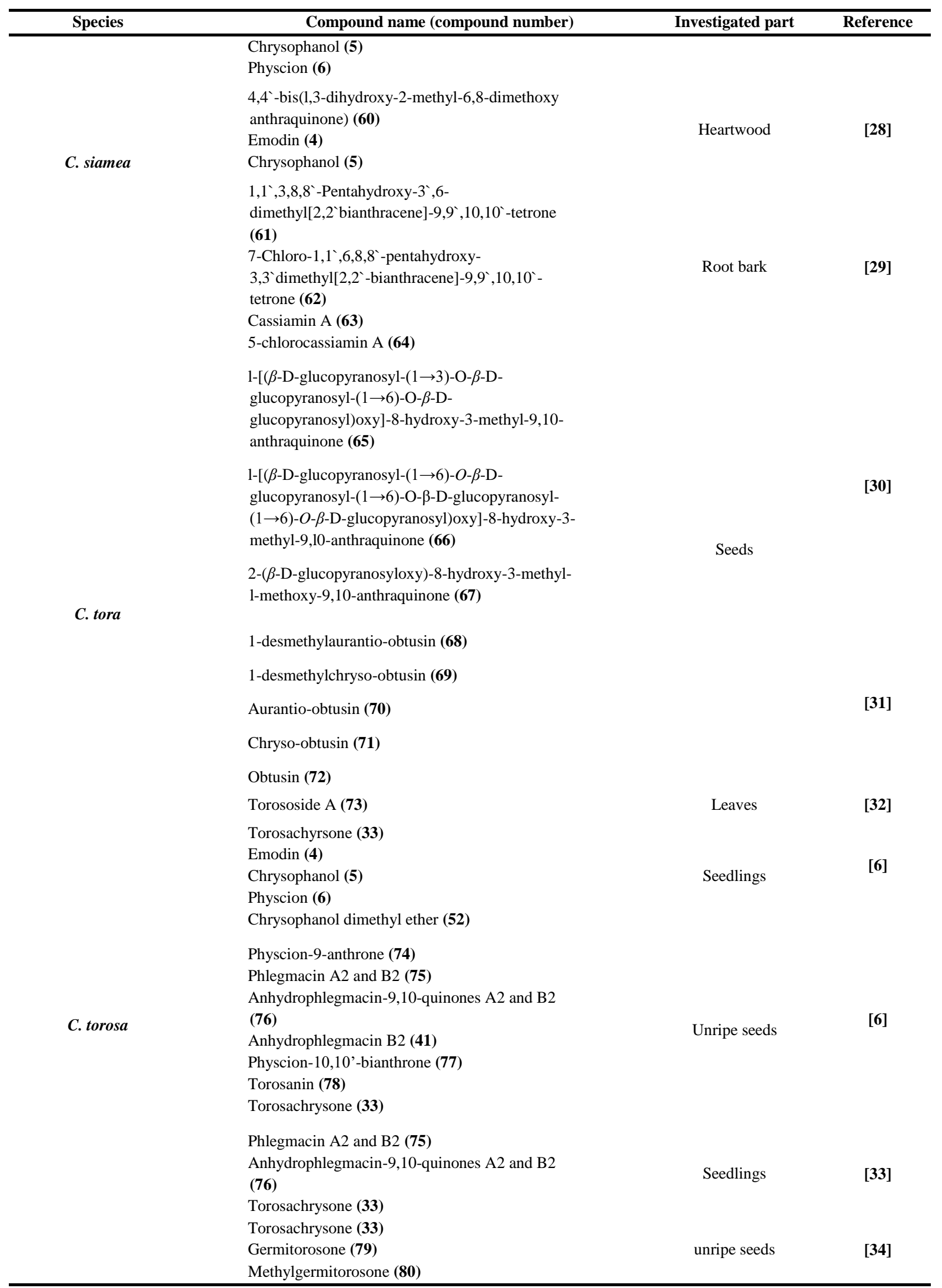


Table 2. Phenolic compounds reported in different Cassia species

\begin{tabular}{|c|c|c|c|}
\hline Species & Compound name (compound number) & Investigated part & Reference \\
\hline \multirow[t]{2}{*}{ C. alata } & $\begin{array}{l}\text { Chrysoeriol-7-0-(2“- } O-\beta \text {-D-mannopyranosyl)- } \beta \text {-D- } \\
\text { allopyranoside (81) } \\
\text { Rhamnetin-3-0-(2“-O- } O \text {-D-mannopyranosyl)- } \beta \text {-D- } \\
\text { allopyranoside (82) }\end{array}$ & Seeds & [35] \\
\hline & Kampferol (83) & Root & [9] \\
\hline C. auriculata & $\begin{array}{l}\text { Kampferol (83) } \\
\text { Quercetin (84) } \\
\text { Kaempferol 3-O-rutinoside (85) } \\
\text { Luteolin (86) }\end{array}$ & Aerial parts & [36] \\
\hline C. angustifolia & $\begin{array}{l}\text { Quercetine 3-O-gentiobioside (87) } \\
\text { Kaempferol 3-O-gentiobioside (88) } \\
\text { Isorhamentin 3-O-gentiobiosid (89) }\end{array}$ & Leaves & [37] \\
\hline C. fistula & $\begin{array}{l}\text { (2`S)-7-hydroxy-5-hydroxymethyl-2-(2`-hydroxypropyl) } \\
\text { chromone (90) } \\
\text { Benzyl-2-O- } \beta \text {-D-glucopyranosyl-3,6- } \\
\text { dimethoxybenzoate } \mathbf{9 1 )}\end{array}$ & Leaves & [38] \\
\hline \multirow[t]{2}{*}{ C. glauca } & $\begin{array}{l}\text { Apigenin (92) } \\
\text { Luteolin } \mathbf{( 8 6 )} \\
\text { Quercetin }(\mathbf{8 4}) \\
\text { Quercetin-3- } O-\beta \text {-D-glucopyranoside } \mathbf{( 9 3 )} \\
\text { Kaempferol-3- } O \text {-rutinoside (85) } \\
\text { Rutin (94) }\end{array}$ & Leaves & [39] \\
\hline & Quercetin-3-O-glucoside-7- $O$-rahmnoside (95) & & {$[40]$} \\
\hline C. garrettiana & $\begin{array}{l}\text { Cassigarol A (96) } \\
\text { Cassigarol A (96) } \\
\text { Cassigarol C (97) } \\
\text { Cassigarol B (98) } \\
\text { Cassigarol D (99) } \\
\text { Cassigarol E (100) } \\
\text { Cassigarol F (101) } \\
\text { Cassigarol G (102) }\end{array}$ & Heart wood & $\begin{array}{l}{[41]} \\
{[42]}\end{array}$ \\
\hline C. italic & 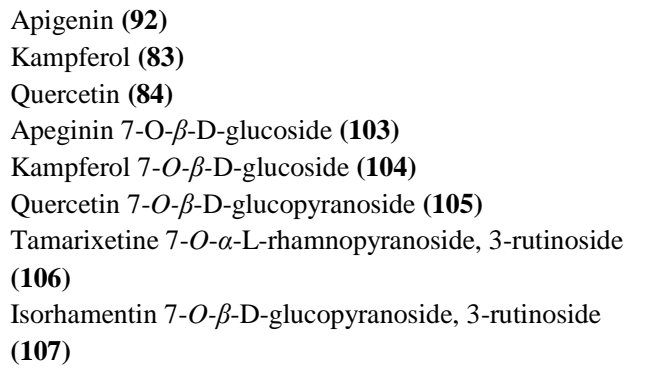 & Aerial parts & [44] \\
\hline C. javanica & $\begin{array}{l}\text { Dihydrorhamnetin-3-O- } \beta \text {-D-glucopyranoside (108) } \\
\text { Leucocyanidin- } 4 \text {-O-methyl ether-3-O- } \beta \text {-D- } \\
\text { galactopyranoside (109) }\end{array}$ & Flowers & [45] \\
\hline C. nomame & $\begin{array}{l}\text { Demethyltorosaflavone C (110) } \\
\text { Demethyltorosaflavone D (111) } \\
\text { Luteolin (86) } \\
\text { Luteolin 7- } O-\beta \text {-D-glucopyranoside (112) } \\
\text { Vitexin (113) }\end{array}$ & Aerial parts & {$[46]$} \\
\hline
\end{tabular}




\begin{tabular}{|c|c|c|c|}
\hline Species & Compound name (compound number) & Investigated part & Reference \\
\hline C. obtusifolia & $\begin{array}{l}\text { 2-benzyl-4,6-dihydroxy benzoic acid (114) } \\
\text { 2-benzyl-4,6-dihydroxy benzoic acid-6-O- } \beta \text {-D- } \\
\text { glucopyranoside (115) } \\
\text { 2-benzyl-4,6-dihydroxy benzoic acid-4-O- } \beta \text {-D- } \\
\text { glucopyranoside (116) } \\
\text { 2-benzyl-4,6-dihydroxy benzoic acid-6- } O-\left[2^{\prime}, 6 `-O \text { - }\right. \\
\text { diacetyl]- } \beta \text {-D-glucopyranoside (117) } \\
\text { 2-benzyl-4,6-dihydroxy benzoic acid-6- } O-\left[3^{\prime}, 6-O \text { - }\right. \\
\text { diacetyl]- } \beta \text {-D-glucopyranoside (118) } \\
\text { 2-benzyl-4,6-dihydroxy benzoic acid-6- } O-\left[4^{\prime}, 6-O-\right. \\
\text { diacetyl]- } \beta \text {-D-glucopyranoside (119) }\end{array}$ & Seeds & {$[47]$} \\
\hline C. torosa & $\begin{array}{l}\text { 2,3-dihydro-8,10-dihydtoxy-2,5-dimethyl-4H- } \\
\text { naphtho[1,2-b]pyran-4-one (120) }\end{array}$ & Unripe seeds & [34] \\
\hline
\end{tabular}

Table 3. Some miscellaneous compounds have been isolated from different Cassia species

\begin{tabular}{|c|c|c|c|}
\hline Species & Compound name (compound number) & Investigated part & Reference \\
\hline \multirow[t]{2}{*}{ C. auriculata } & & Leaves & {$[48]$} \\
\hline & Di-(2-ethyl)-hexylphthalate (121) & & \\
\hline \multirow[t]{2}{*}{ C. floribunda } & & Leaves & [49] \\
\hline & $\mathrm{N}^{1}, \mathrm{~N}^{8}$-dibenzoylspermidine (122) & & \\
\hline \multirow[t]{8}{*}{ C. glauca } & Palmitic acid (123) & Seeds oil & [50] \\
\hline & Stearic acid (124) & & \\
\hline & Oleic acid (125) & & \\
\hline & Linoleic acid (126) & & \\
\hline & Linolenic acid (127) & & \\
\hline & Arachidic acids (128) & & \\
\hline & $\beta$-Sitosterol- $\beta$-D- glucoside (129) & Stem & [15] \\
\hline & Galactomannan (130) & & [6] \\
\hline \multirow[t]{2}{*}{ C. italic } & $\beta$-Sitosterol (131) & Leaves & [51] \\
\hline & Stigmasterol (132) & & \\
\hline \multirow[t]{9}{*}{ C. laevigata } & Calendin (133) & Leaves & {$[52]$} \\
\hline & Cinnamic acid (134) & & \\
\hline & $\begin{array}{l}\text { 3-hydroxy-1-(4-hydroxy-3-methoxyphenyl)-propan-1- } \\
\text { one (135) }\end{array}$ & & \\
\hline & 2,3-dihydroxy-1-(4-hydroxy-3-methoxyphenyl)-propan- & & \\
\hline & 1 -one (136) & & \\
\hline & 3-hydroxy-1- (4-hydroxy-3,5-dimethoxyphenyl)-propan- & & \\
\hline & 1-one (137) & & \\
\hline & Syringic acid (138) & & \\
\hline & Vanillic acid (139) & & \\
\hline \multirow[t]{8}{*}{ C. leptophylla } & $(-)$-spectalin $(\mathbf{1 4 0})$ & Leaves & [53] \\
\hline & (-)-spectalinin (141) & & \\
\hline & Canavalin (142) & & \\
\hline & Leptophyllin A (143) & & \\
\hline & 3-acetylleptophyllin (144) & & \\
\hline & (+)-spectaline (145) & & \\
\hline & Iso-6-canavaline (146) & & \\
\hline & Leptophyllin B (147) & & \\
\hline
\end{tabular}




\begin{tabular}{|c|c|c|c|}
\hline Species & Compound name (compound number) & Investigated part & Reference \\
\hline C. multijuga & $\begin{array}{l}\text { 5-acetonyl-7- hydroxy-2-methylchromone (148) } \\
5 \text { - Acetonyl - } 2 \text { - methylchromone - } 7 \text { - } O \text { - } \beta \text { - D- } \\
\text { glucopyranoside (149) }\end{array}$ & Leaves & [54] \\
\hline C. nodosa & Nodolidate (150) & Bark & [55] \\
\hline C. occidentalis & Phytosterol (151) & Roots & [21] \\
\hline C. obtusifolia & $\begin{array}{l}(4 \mathrm{R}, 5 \mathrm{~S}, 6 \mathrm{E}, 8 \mathrm{Z}) \text {-ethyl-4-[(E)-but-1-enyl]-5- } \\
\text { hydroxypentadeca-6,8- dionate (152) } \\
\text { Stigmasterol (132) } \\
\text { Lupeol (153) } \\
\text { Buteolinic acid (154) } \\
\text { Friedelin (155) }\end{array}$ & Leaves & [22] \\
\hline C. sophera & $\beta$-Sitosterol (131) & Leaves & {$[56]$} \\
\hline C. tora & Cassitoroside (156) & Seeds & [57] \\
\hline \multirow[t]{3}{*}{ C. siamea } & Barakol (157) & Leaves & {$[58]$} \\
\hline & $\begin{array}{l}\text { Chrobisiamone A (158) } \\
\text { Cassiarin A (159) }\end{array}$ & & [59] \\
\hline & $\begin{array}{l}\text { Cassiadinine (160) } \\
\text { 5-acetonyl-7- hydroxy-2-methylchromone (148) } \\
(+)-6-H y d r o x y m e l l e i n ~(\mathbf{1 6 1}) \\
\text { (+)-6-Hydroxymellein diacetate (162) } \\
\text { Chaksine (163) }\end{array}$ & Flowers & {$[60]$} \\
\hline
\end{tabular}

Table 4. Biological activities of genus Cassia

\begin{tabular}{|c|c|c|c|}
\hline Biological activities & Species & $\begin{array}{l}\text { Part or compound responsible for } \\
\text { the activity }\end{array}$ & Reference \\
\hline Antacid activity & C. garrttiana & Cassigarol A (Heartwood) & [41] \\
\hline \multirow{9}{*}{ Anxiolytic activity } & C. siamea & Barakol (leaves) & {$[60]$} \\
\hline & C. abbreviata & Root methanol extract & {$[61]$} \\
\hline & & Kampferol & \\
\hline & & Quercetin & {$[36]$} \\
\hline & & Kaempferol 3-O-rutinoside & {$[36]$} \\
\hline & C. auriculata & $\begin{array}{l}\text { Luteolin } \\
\text { (Aerial parts) }\end{array}$ & \\
\hline & & Flowers extract & [62] \\
\hline & & Different root fractions & [63] \\
\hline & C. angustifolia & Aqueous flower extract & {$[64]$} \\
\hline \multirow{10}{*}{ Antioxidant activity } & & $\begin{array}{l}\text { Crude extracts from stem bark, } \\
\text { leaves, flowers, and fruit pulp }\end{array}$ & {$[65]$} \\
\hline & C. fistula & Flower butanol extract & {$[66]$} \\
\hline & & Leave ethanol extract & $\begin{array}{l}{[67]} \\
{[68]}\end{array}$ \\
\hline & & Leaves methanol extract & {$[60]$} \\
\hline & & Leaves ethanol extract & {$[70],[71]$} \\
\hline & & Flower methanol extract & [72] \\
\hline & C. glauca & $\begin{array}{l}\text { Leaves, flower, stem and pod } \\
\text { methanol extracts }\end{array}$ & [73] \\
\hline & & Seed extract & [74] \\
\hline & & Acetone seed extract & [75] \\
\hline & & Acetone seed extract & [71] \\
\hline
\end{tabular}




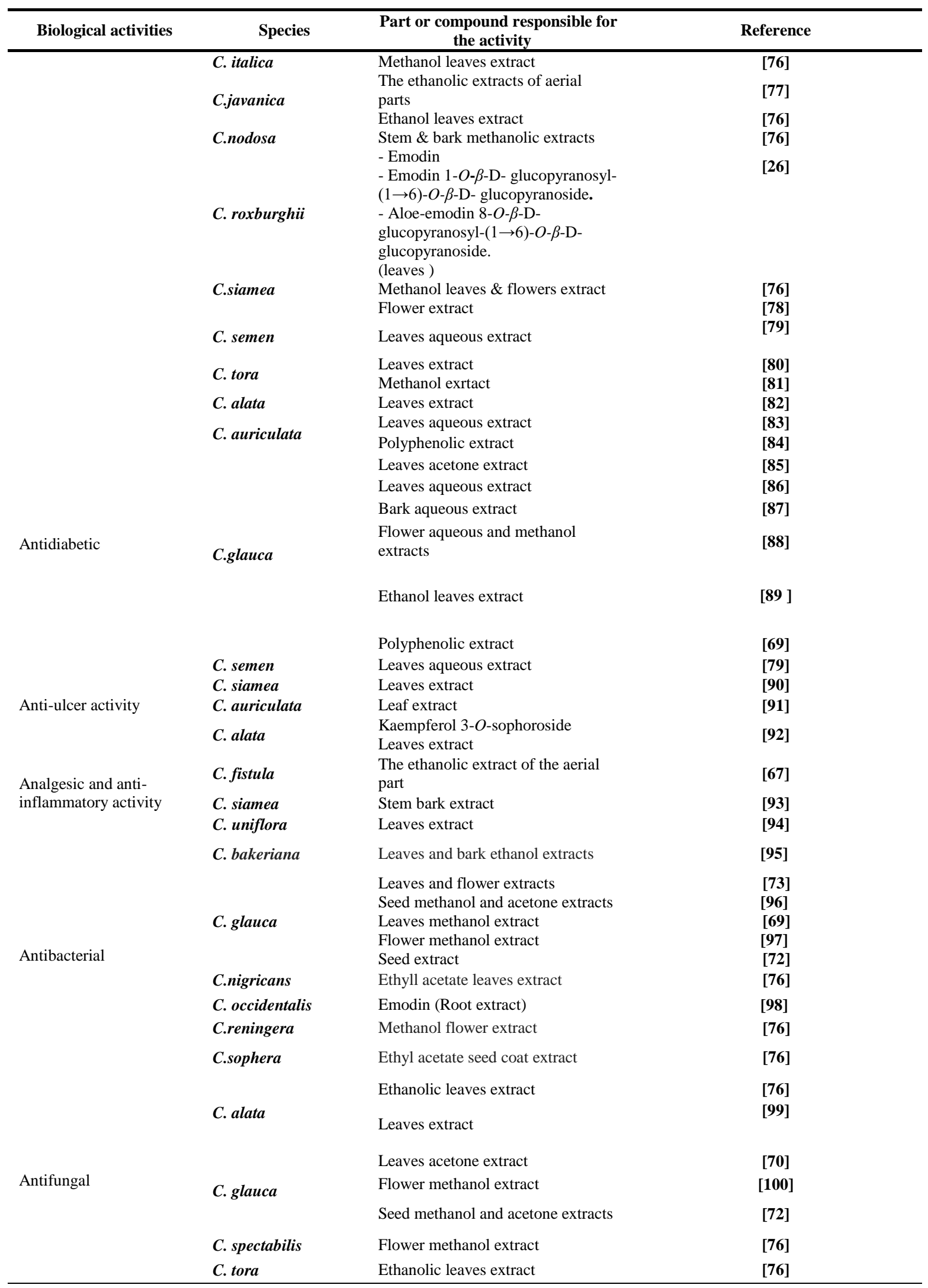




\begin{tabular}{|c|c|c|c|}
\hline Biological activities & Species & $\begin{array}{l}\text { Part or compound responsible for } \\
\text { the activity }\end{array}$ & Reference \\
\hline \multirow{5}{*}{ Cytotoxic activity } & \multirow{2}{*}{$\begin{array}{l}\text { C. bakeriana } \\
\text { C. fistula }\end{array}$} & Leaves and bark ethanol extracts & {$[95]$} \\
\hline & & Leaves extract & [101] \\
\hline & \multirow{2}{*}{ C. glauca } & Leaves ethanol extract & [69] \\
\hline & & Leaves methanol extract & {$[68]$} \\
\hline & C. tora & $\begin{array}{l}\text { Hydro alcoholic extract of aerial } \\
\text { part }\end{array}$ & [102] \\
\hline \multirow{5}{*}{ Hypolipidemic } & \multirow{2}{*}{ C. auriculata } & Leaves aqueous extract & [83] \\
\hline & & Flowers extract & [103] \\
\hline & C. siamea & Leaves extract & [78] \\
\hline & C. semen & Leaves aqueous extract & [79] \\
\hline & C. tora & Seed extract & {$[104]$} \\
\hline \multirow{7}{*}{ Hepatoprotective activity } & C. abbreviata & Root methanol extract & [61] \\
\hline & C. auriculata & Flower ethanol extract & [106] \\
\hline & C. fistula & Leaves extract & [107] \\
\hline & \multirow{2}{*}{ C. glauca } & Leaves methanol extract & [108] \\
\hline & & Leaves extract & {$[68]$} \\
\hline & C. occidentalis & Leaves methanol extract & [109] \\
\hline & C. auriculata & Flower ethanol extract & {$[105]$} \\
\hline $\begin{array}{l}\text { Immunomodulatory } \\
\text { activity }\end{array}$ & C. auriculata & $\begin{array}{l}\text { Polyphenols derived from flowers } \\
\text { extract }\end{array}$ & [110] \\
\hline Laxative activity & $\begin{array}{l}\text { C. alata } \\
\text { C. podocarpa }\end{array}$ & Leaves extract & [111] \\
\hline Wound healing & C. fistula & Leaves extract & [112] \\
\hline
\end{tabular}<smiles>COc1cc(O)c2c(O)c3c(cc2c1)CC(O)(O)CC3=O</smiles>

Fig.1.1. Structure of compound 1

R1

(2)

(3)
$\mathrm{OH}$

$\mathrm{OH}$

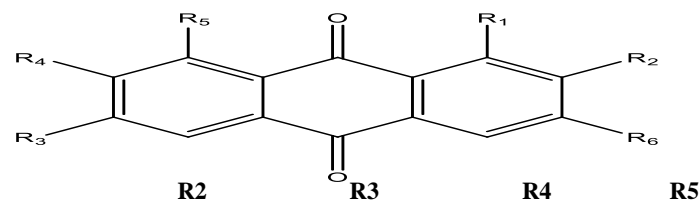

$\mathrm{H}$

$\mathrm{H}$

$\mathrm{H}$

$\mathrm{OH}$

$\mathrm{OH}$

$\mathrm{H}$

$\mathrm{H}$

$\mathrm{H}$

$\mathrm{H}$

Fig.1.2. Structure of compounds 2 and 3<smiles>[Y5]c1cc([R16])c2c(c1[R20])C(=O)c1c(OCC)cc(C)cc1C2=O</smiles>

$\mathbf{R}_{1}$

(4)

(5)

(6)
H

$\mathrm{H}$

$\mathrm{H}$
$\mathbf{R}_{3}$

$\mathrm{OH}$

H

$\mathrm{OMe}$
$\mathbf{R}_{4}$

$\mathrm{H}$

$\mathrm{H}$

H

Fig. 1.3. Structure of compounds $4-6$ 


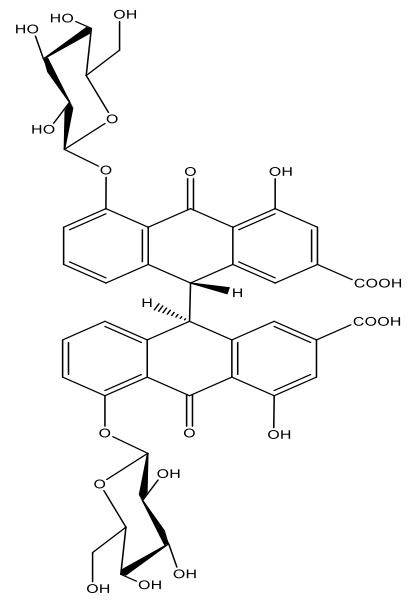

(7)

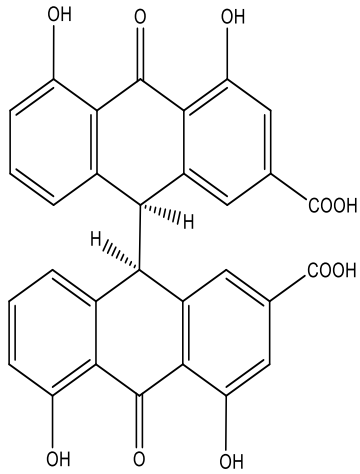

(10)

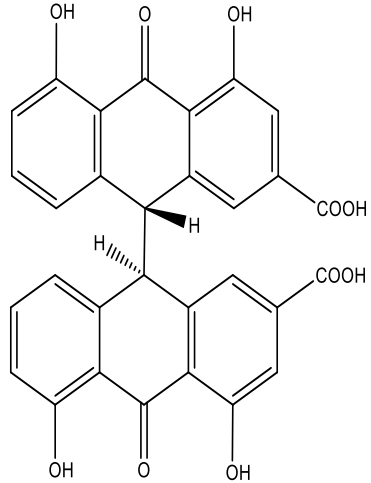

(8)

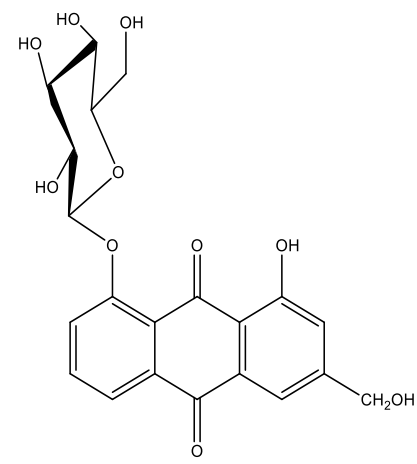

(11)

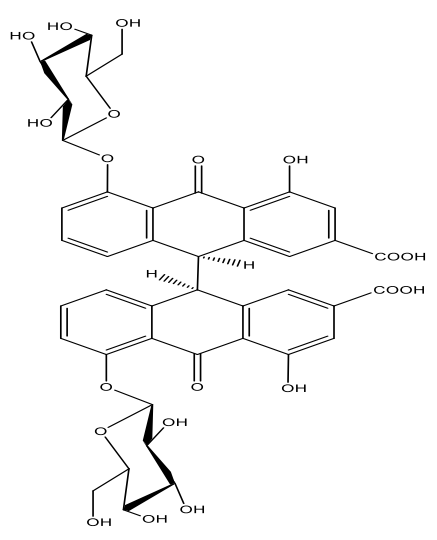

(9)

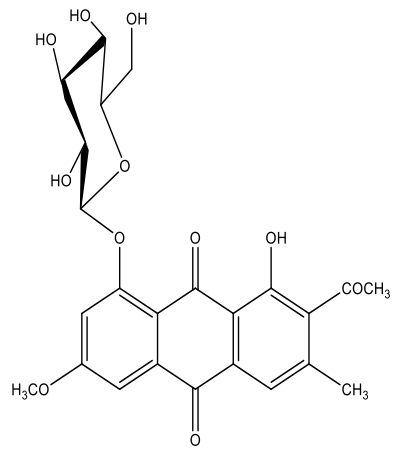

(12)

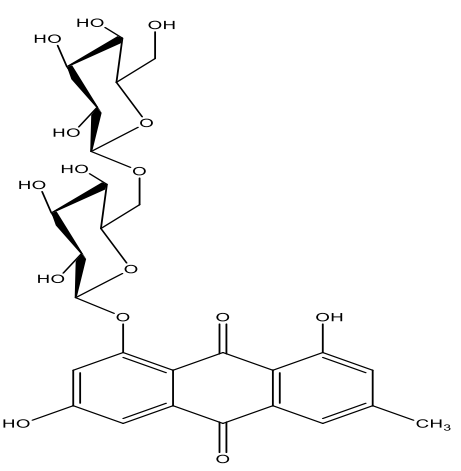

(13)

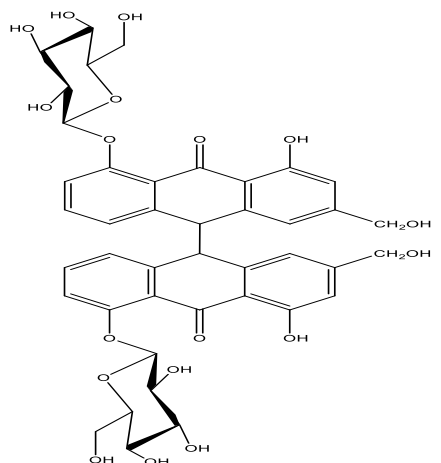

(14)

Fig. 1.4. Structure of compounds $4-14$ 

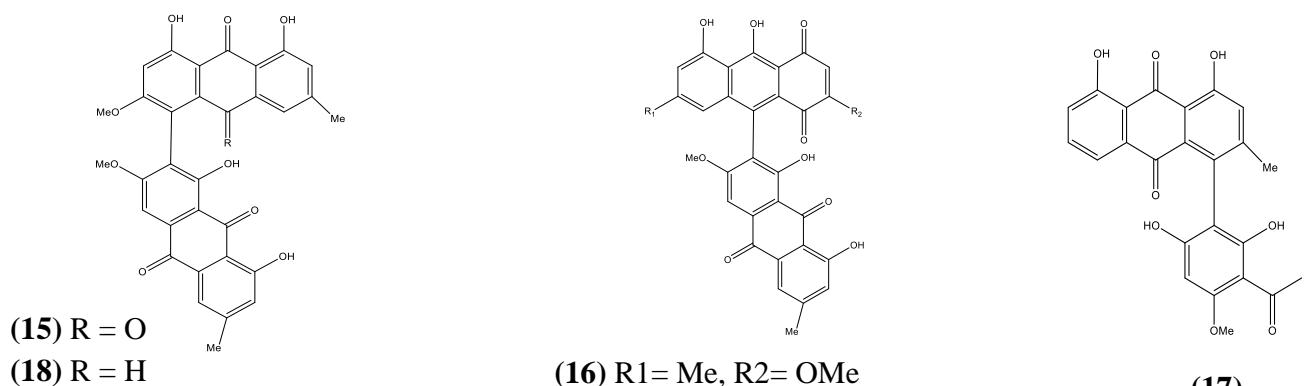

(16) $\mathrm{R} 1=\mathrm{Me}, \mathrm{R} 2=\mathrm{OMe}$

(17)

Fig. 1.5. Structure of compounds $15-18$<smiles>[R9]c1cc2c(c([R3])c1[R3])C(=O)c1c([R3])cc([R9])c([R3])c1C2=O</smiles>

$\mathbf{R}_{1}$

(19)

(20)

(21)

(22)

(23)

(24)

(25)

$\mathrm{OH}$

$\mathrm{OH}$

$\mathrm{H}$
$\mathrm{OMe}$

$\mathrm{OMe}$

$\mathrm{CH}_{2} \mathrm{OH}$

$\mathrm{OMe}$

$\mathbf{R}_{\mathbf{2}}$

$\mathrm{Me}$

$\mathrm{Me}$

$\mathrm{Me}$

$\mathrm{Me}$

$\mathrm{OMe}$

$\mathrm{Me}$

$\mathrm{Me}$
$\mathbf{R}_{3}$

$\mathrm{OMe}$

$\mathrm{OMe}$

$\mathrm{OMe}$

OMe

$\mathrm{OMe}$

$\mathrm{OH}$

$\mathrm{OMe}$
$\mathbf{R}_{4}$

$\mathrm{OH}$

$\mathrm{OH}$

$\mathrm{OH}$<smiles>COCOC</smiles>

$\mathrm{OH}$

$\mathrm{OH}$

$\mathrm{OH}$

Fig. 1.6. Structure of compounds 19 - 25<smiles>[Y9]c1c([13CH3])cc(OCC)c2c1C(=O)c1cc([N+](=O)[O-])cc(OCC)c1C2=O</smiles>

$\mathbf{R}_{1}$

$\mathbf{R}_{\mathbf{2}}$

$\mathbf{R}$

O- $\alpha$-L-rhamnopyranoside

$\mathrm{OH}$

$\mathrm{OH}$

$\mathrm{OH}$

Fig. 1.7. Structure of compounds 26 and 27 


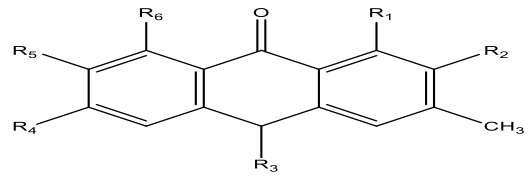

$\begin{array}{ccccccc} & \mathbf{R}_{\mathbf{1}} & \mathbf{R}_{\mathbf{2}} & \mathbf{R}_{\mathbf{3}} & \mathbf{R}_{\mathbf{4}} & \mathbf{R}_{\mathbf{5}} & \mathbf{R}_{\mathbf{6}} \\ \text { (28) } & \mathrm{OH} & \mathrm{H} & \mathrm{OCO}\left(\mathrm{CH}_{2}\right)_{8} \mathrm{CH}_{3} & \mathrm{OMe} & \mathrm{H} & \mathrm{OH} \\ \text { (29) } & \mathrm{OH} & \mathrm{H} & \mathrm{OCO}\left(\mathrm{CH}_{2}\right)_{12} \mathrm{CH}_{3} & \mathrm{H} & \mathrm{H} & \mathrm{OH} \\ \text { (30) } & \mathrm{OH} & \mathrm{H} & \mathrm{OCO}\left(\mathrm{CH}_{2}\right)_{14} \mathrm{CH}_{3} & \mathrm{H} & \mathrm{H} & \mathrm{OH} \\ \text { (31) } & \mathrm{OMe} & \mathrm{OH} & \mathrm{OCO}\left(\mathrm{CH}_{2}\right)_{8} \mathrm{CH}_{3} & \mathrm{OH} & \mathrm{OH} & \mathrm{OMe}\end{array}$

Fig. 1.8. Structure of compounds 28 - 31

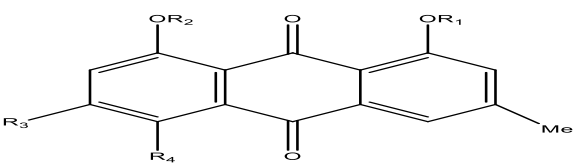

$\mathbf{R}_{1}$

(32) $\mathrm{H}$
$\mathbf{R}_{3} \quad \mathbf{R}_{4}$

$\mathrm{OMe} \quad \mathrm{H}$

Fig. 1.9. Structure of compound 32<smiles>COc1cc(O)c2c(O)c3c(cc2c1)CC(C)(O)CC3=O</smiles>

(33)
(34) $\mathrm{R}=\mathrm{H}$

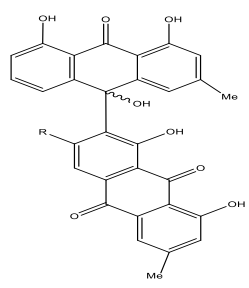

Fig. 1.10. Structure of compounds 33 and 34
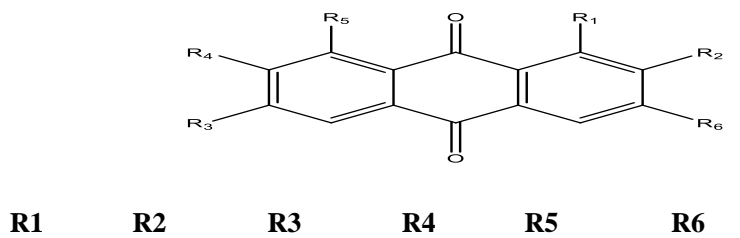

(35)

$\begin{array}{lllllll} & \mathrm{OH} & \mathrm{H} & \mathrm{H} & \mathrm{OH} & \mathrm{OH} & \mathrm{Me}\end{array}$

Fig. 1.11. Structure of compound 35 

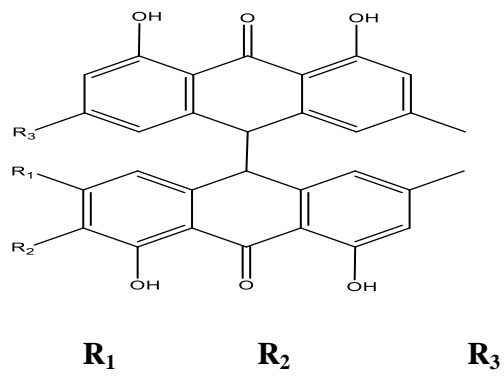

$\begin{array}{cccc}\text { (36) } & \mathrm{H} & \mathrm{H} & \mathrm{H} \\ \text { (37) } & \mathrm{OMe} & \mathrm{H} & \mathrm{H} \\ \text { (38) } & \mathrm{H} & \mathrm{OMe} & \mathrm{H} \\ \text { (39) } & \mathrm{H} & \mathrm{OMe} & \mathrm{OMe}\end{array}$

Fig. 1.12. Structure of compounds 36 - 39
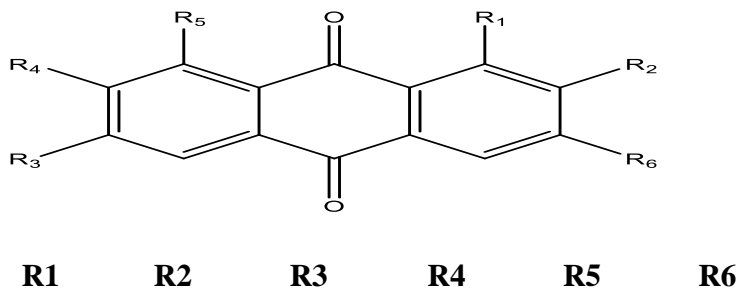

(40) $\begin{array}{llllll}\mathrm{OH} & \mathrm{Me} & \mathrm{OMe} & \mathrm{H} & \mathrm{OMe} & \mathrm{R}\end{array}$

Fig. 1.13. Structure of compound 40

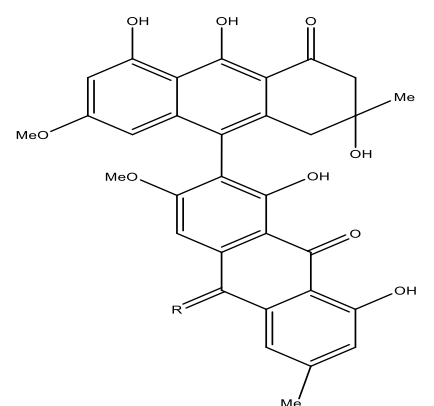

(41) $\mathrm{R}=\mathrm{H}$

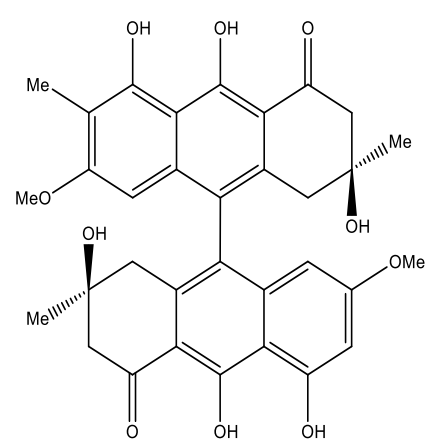

(44)

Fig. 1.14. Structure of compounds 41 and 44 


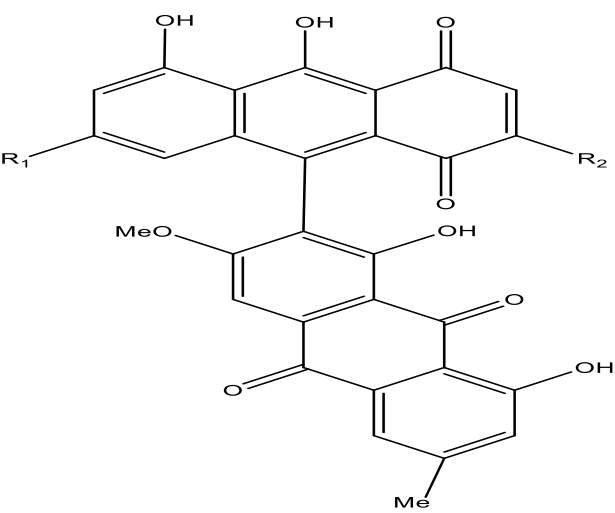

$\mathbf{R}_{1}$

(42)

(43)
$\mathrm{OMe}$

H
$\mathbf{R}_{2}$

Me

Me

Fig. 1.15. Structure of compounds 42 and 43

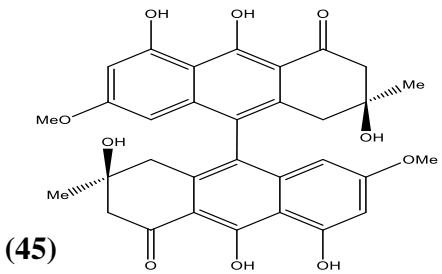

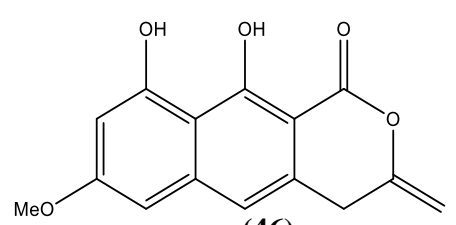

(46)

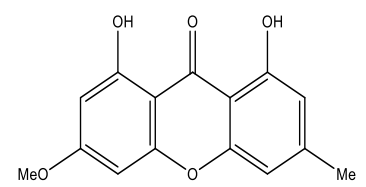

(49)
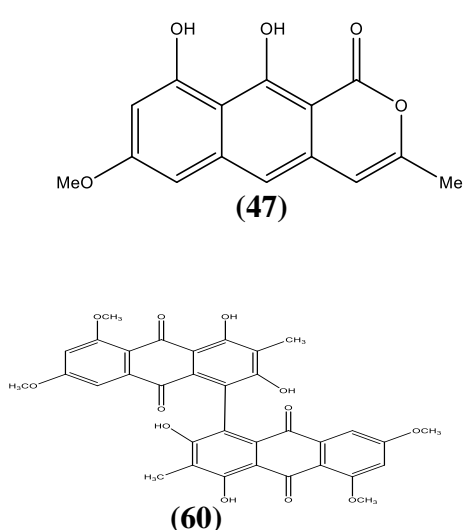

Fig. 1.16. Structure of compounds $45-49$ and 60 


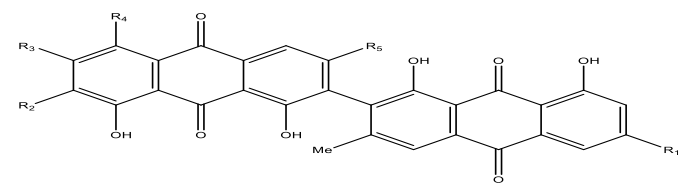

(61)

$\mathbf{R}_{1}$

$\mathbf{R}_{2}$

$\mathbf{R}_{3}$

$\mathbf{R}_{4}$

$\mathbf{R}_{5}$

(62)

$\mathrm{H}$

$\mathrm{H}$

$\mathrm{Me}$

$\mathrm{H}$

$\mathrm{OH}$

$\mathrm{H}$

$\mathrm{Cl}$

$\mathrm{OH}$

H

$\mathrm{Me}$

(63)

$\mathrm{H}$

$\mathrm{H}$

$\mathrm{OH}$

$\mathrm{H}$

$\mathrm{Me}$

(64)

$\mathrm{H}$

$\mathrm{H}$

$\mathrm{OH}$

$\mathrm{Cl}$

Me

Fig. 1.17. Structure of compounds $61-64$

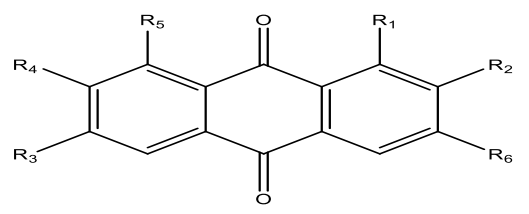

\begin{tabular}{|c|c|c|c|c|c|c|}
\hline & $\mathbf{R} 1$ & $\mathbf{R 2}$ & $\mathbf{R 3}$ & R4 & $\mathbf{R 5}$ & R6 \\
\hline$(\mathbf{5 0})$ & $\mathrm{OH}$ & $\mathrm{H}$ & $\mathrm{OMe}$ & $\mathrm{Me}$ & $\mathrm{H}$ & $\mathrm{OH}$ \\
\hline (51) & $\mathrm{OH}$ & $\mathrm{H}$ & $\mathrm{H}$ & $\mathrm{CHO}$ & $\mathrm{H}$ & $\mathrm{CHO}$ \\
\hline (53) & $\mathrm{R}$ & $\mathrm{H}$ & $\mathrm{OH}$ & $\mathrm{H}$ & $\mathrm{OH}$ & $\mathrm{Me}$ \\
\hline (54) & $\mathrm{R}$ & $\mathrm{H}$ & $\mathrm{H}$ & $\mathrm{H}$ & $\mathrm{OH}$ & $\mathrm{CH}_{2} \mathrm{OH}$ \\
\hline (57) & $\mathrm{OH}$ & $\mathrm{OH}$ & $\mathrm{OH}$ & $\mathrm{OMe}$ & $\mathrm{OMe}$ & $\mathrm{Me}$ \\
\hline (58) & $\mathrm{OH}$ & $\mathrm{OH}$ & $\mathrm{OMe}$ & $\mathrm{OH}$ & $\mathrm{OMe}$ & $\mathrm{Me}$ \\
\hline (59) & $\mathrm{OH}$ & $\mathrm{Me}$ & $\mathrm{H}$ & $\mathrm{H}$ & $\mathrm{OH}$ & Neohesperidoside \\
\hline (68) & $\mathrm{OH}$ & $\mathrm{OH}$ & $\mathrm{OH}$ & $\mathrm{OMe}$ & $\mathrm{OH}$ & $\mathrm{Me}$ \\
\hline (69) & $\mathrm{OH}$ & $\mathrm{OH}$ & $\mathrm{OMe}$ & $\mathrm{OMe}$ & $\mathrm{OMe}$ & $\mathrm{Me}$ \\
\hline (70) & $\mathrm{OMe}$ & $\mathrm{OH}$ & $\mathrm{OH}$ & $\mathrm{OMe}$ & $\mathrm{OH}$ & $\mathrm{Me}$ \\
\hline (71) & $\mathrm{OMe}$ & $\mathrm{OH}$ & $\mathrm{OMe}$ & $\mathrm{OMe}$ & $\mathrm{OMe}$ & $\mathrm{Me}$ \\
\hline (72) & $\mathrm{OMe}$ & $\mathrm{OH}$ & $\mathrm{OMe}$ & $\mathrm{OMe}$ & $\mathrm{OH}$ & $\mathrm{Me}$ \\
\hline
\end{tabular}

Fig. 1.18. Structure of compounds $50-54,57-59$ and 68-72 

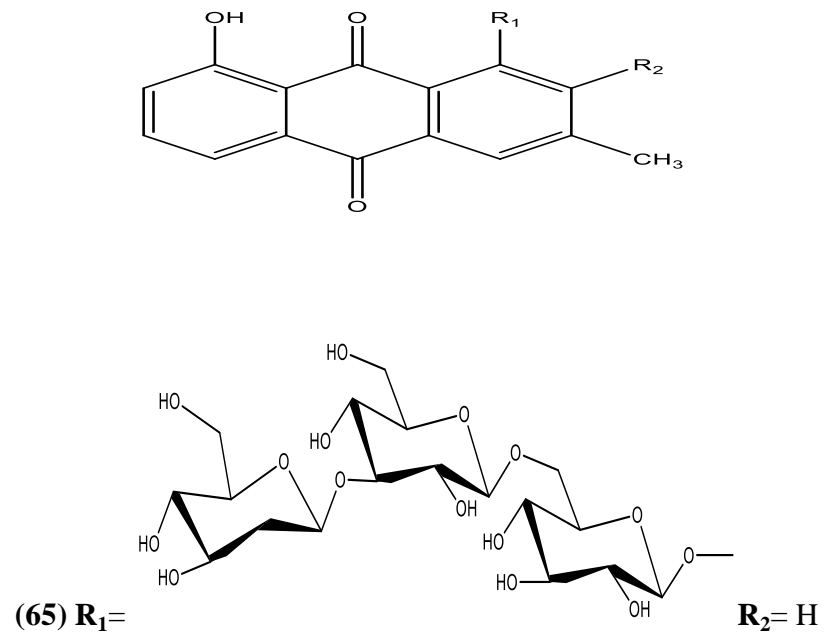

(66) $\mathbf{R}_{1}=$

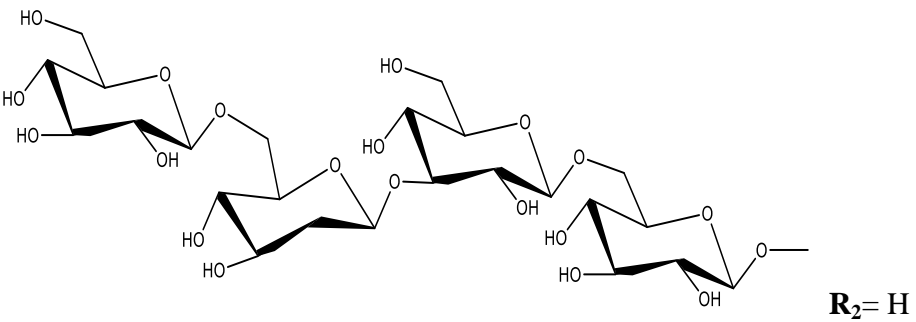

(67) $\mathbf{R}_{\mathbf{1}}=\mathrm{OCH}_{3} \quad \mathbf{R}_{\mathbf{2}}=\beta$-D-glucopyranoside

Fig. 1.19. Structure of compounds $65-67$

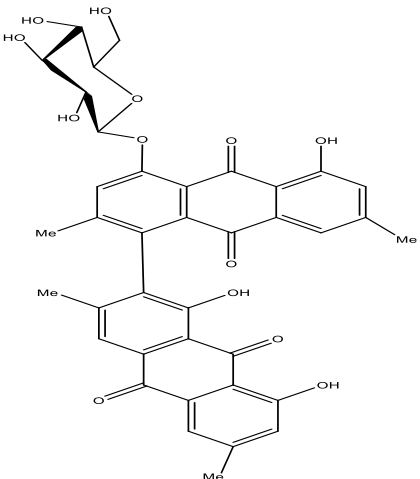

(73)<smiles>C=C1c2cc(O)cc(O)c2C(=O)c2c(O)cc(OC)cc21</smiles>

(74)

Fig. 1.20. Structure of compounds 73 and 74 
<smiles></smiles>

(75)<smiles></smiles>

(78)

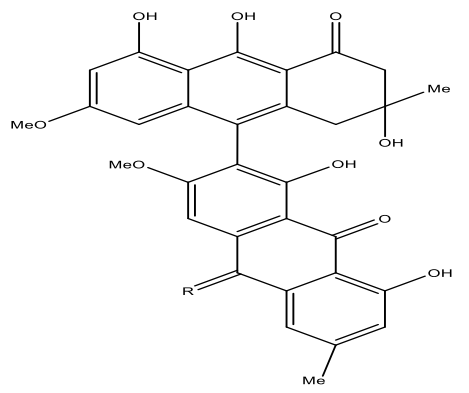

(76) $\mathrm{R}=\mathrm{O}$<smiles>Cc1cc(O)c2c(c1)C(C1c3cc(C)cc(O)c3C(=O)c3c(O)cc(C)cc31)c1cc(C)cc(O)c1C2=O</smiles>

(77)<smiles>[R2]C1=c2c3c([R2])c(OC)cc2=CC2=C(CC([Y])(O)C2)C(=C1O)C(C)(O)CC3=O</smiles>

$\begin{array}{llll} & \mathbf{R}_{\mathbf{1}} & \mathbf{R}_{\mathbf{2}} & \\ \text { (79) } & \mathrm{H} & & \mathrm{Me} \\ \text { (80) } & \mathrm{Me} & & \mathrm{Me}\end{array}$

Fig. 1.21. Structure of compounds $75-80$

Fig. 1. Chemical structure of reported anthraquinones, anthracenes and their derivatives in different Cassia species in Fig. 1 (Fig.1.1- Fig.1.21). 


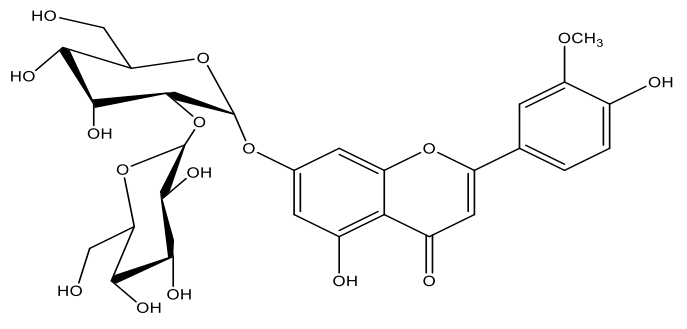

(81)<smiles>CC(O)Cc1cc(=O)c2c(CO)cc(O)cc2o1</smiles>

(90)

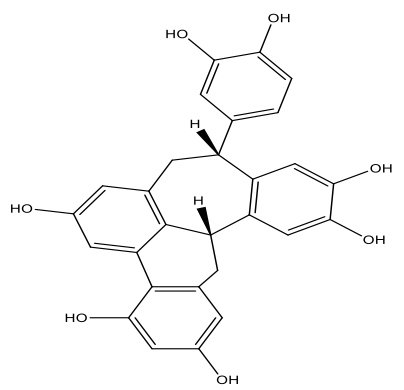

(97)

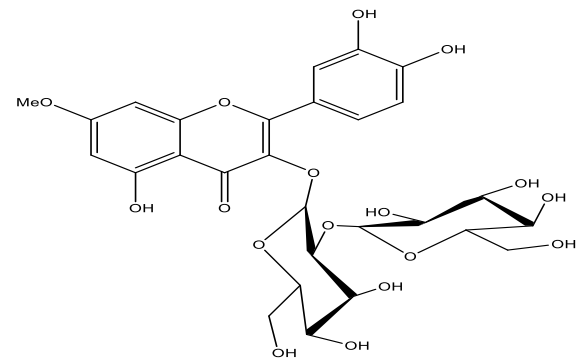

(82)

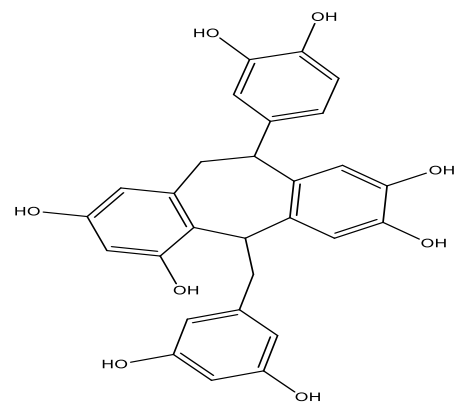

(96)

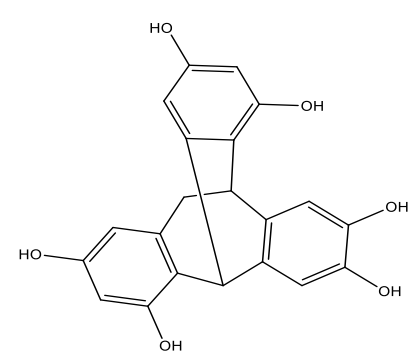

(98)

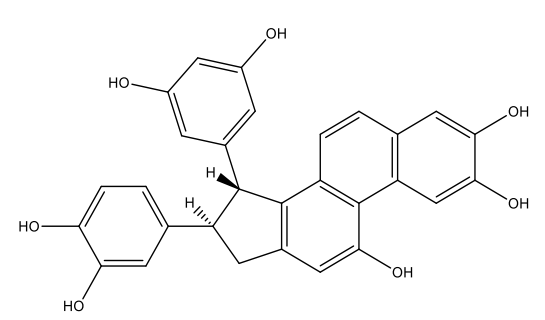

(99)

Fig. 2.1. Structure of compounds $81-82,90-91$ and 96-99

Fig. 2. Chemical structure of reported Phenolic compounds in different Cassia species in figure 2 (Fig.2.1 - Fig.2.5). 


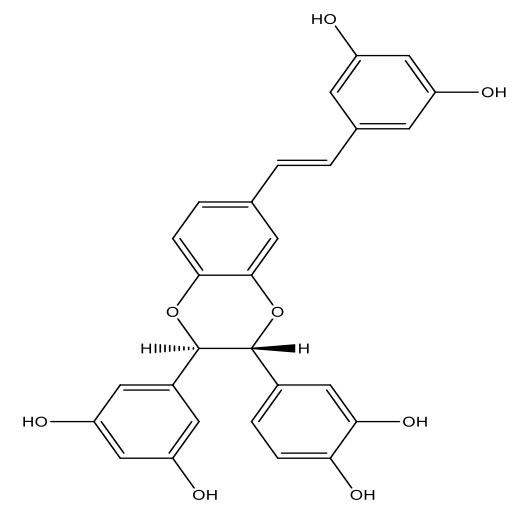

(100) E, (101) Z

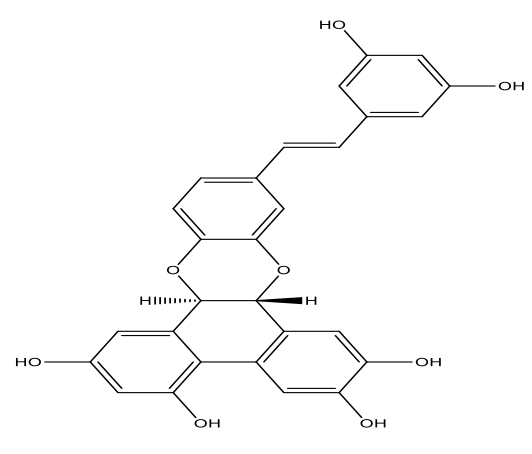

(102)

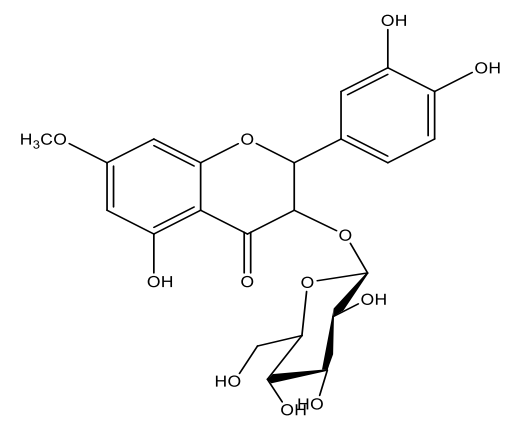

(108)

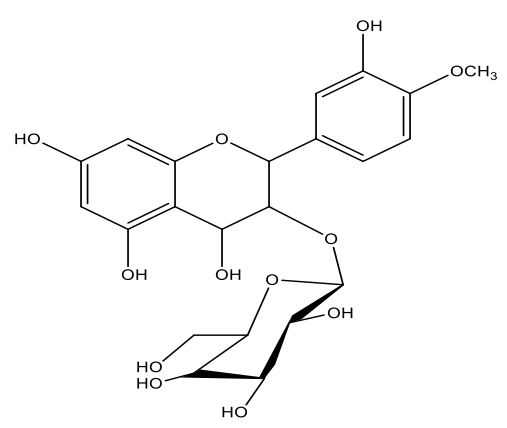

(109)

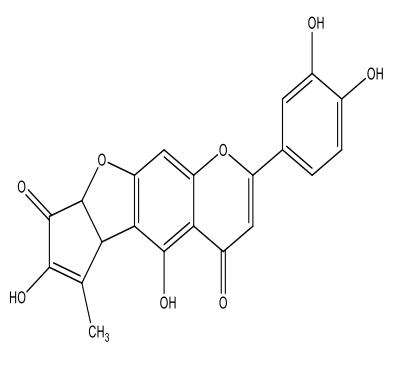

(110)<smiles>O=C(O)/C=C/c1c(O)cc2oc(-c3ccc(O)c(O)c3)cc(=O)c2c1O</smiles>

(111)

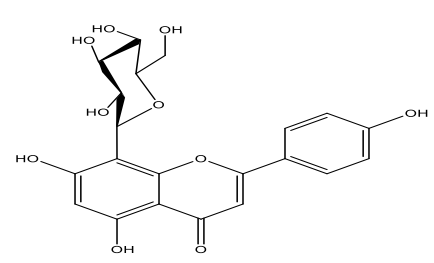

(113)<smiles>O=C(O)c1c(O)cc(O)cc1Cc1ccccc1</smiles>

(114)<smiles>O=C(O)c1c(Cc2ccccc2)cc(O)cc1O[C@@H]1C[C@@H](O)[C@H](O)[C@@H](CO)O1</smiles>

(115)

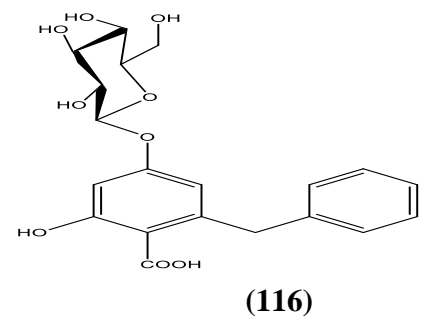

(116)

Fig. 2.2. Structure of compounds 100-102 and 108-116 


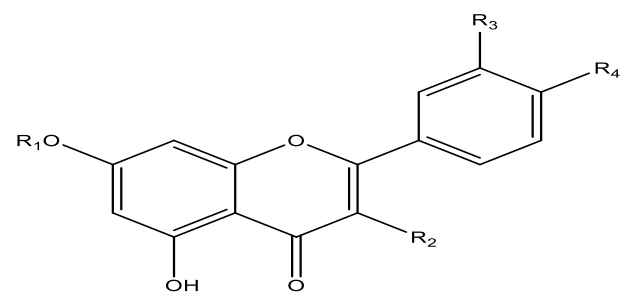

(83)

\begin{tabular}{|c|c|c|c|}
\hline $\mathbf{R 1}$ & $\mathbf{R 2}$ & $\mathbf{R 3}$ & R4 \\
\hline $\mathrm{H}$ & $\mathrm{OH}$ & $\mathrm{H}$ & $\mathrm{OH}$ \\
\hline $\mathrm{H}$ & $\mathrm{OH}$ & $\mathrm{OH}$ & $\mathrm{OH}$ \\
\hline $\mathrm{H}$ & rutinose & $\mathrm{H}$ & $\mathrm{OH}$ \\
\hline $\mathrm{H}$ & $\mathrm{H}$ & $\mathrm{OH}$ & $\mathrm{OH}$ \\
\hline $\mathrm{H}$ & gentiobioside & $\mathrm{OH}$ & $\mathrm{OH}$ \\
\hline $\mathrm{H}$ & gentiobioside & $\mathrm{H}$ & $\mathrm{OH}$ \\
\hline $\mathrm{H}$ & gentiobioside & $\mathrm{OH}$ & $\mathrm{OMe}$ \\
\hline $\mathrm{H}$ & $\mathrm{H}$ & $\mathrm{H}$ & $\mathrm{OH}$ \\
\hline $\mathrm{H}$ & $\beta$-D-glucoside & $\mathrm{OH}$ & $\mathrm{OH}$ \\
\hline $\mathrm{H}$ & rutinoside & $\mathrm{OH}$ & $\mathrm{OH}$ \\
\hline$\alpha$-L-rhamnopyranoside & $\beta$-D-glucoside & $\mathrm{OH}$ & $\mathrm{OH}$ \\
\hline$\beta$-D-glucoside & $\mathrm{H}$ & $\mathrm{H}$ & $\mathrm{OH}$ \\
\hline$\beta$-D-glucoside & $\mathrm{OH}$ & $\mathrm{H}$ & $\mathrm{OH}$ \\
\hline$\beta$-D-glucoside & $\mathrm{OH}$ & $\mathrm{OH}$ & $\mathrm{OH}$ \\
\hline$\alpha$-L-rhamnopyranoside & rutinoside & $\mathrm{OMe}$ & $\mathrm{OH}$ \\
\hline$\beta$-D-glucopyranoside & rutinoside & $\mathrm{OH}$ & $\mathrm{OMe}$ \\
\hline$\beta$-D-glucoside & $\mathrm{H}$ & $\mathrm{OH}$ & $\mathrm{OH}$ \\
\hline
\end{tabular}

Fig. 2.3. Structure of compounds 83-89, 92-95, 103-107 and 112 


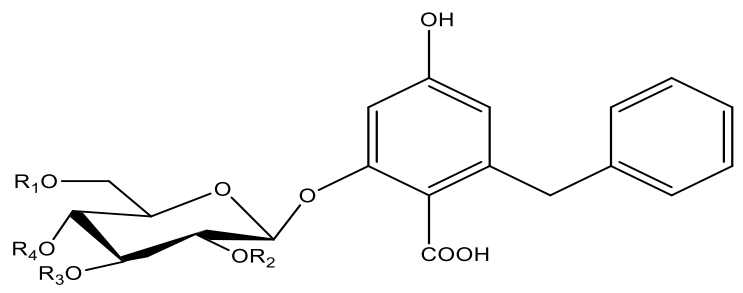

$\begin{array}{ccccc} & \mathbf{R}_{\mathbf{1}} & \mathbf{R}_{\mathbf{2}} & \mathbf{R}_{\mathbf{3}} & \mathbf{R}_{\mathbf{4}} \\ \text { (117) } & \text { Ac } & \text { Ac } & \text { H } & \text { H } \\ \text { (118) } & \text { Ac } & \text { H } & \text { Ac } & \text { H } \\ \text { (119) } & \text { Ac } & \text { H } & \text { H } & \text { Ac }\end{array}$

Fig. 2.4. Structure of compounds 117-119

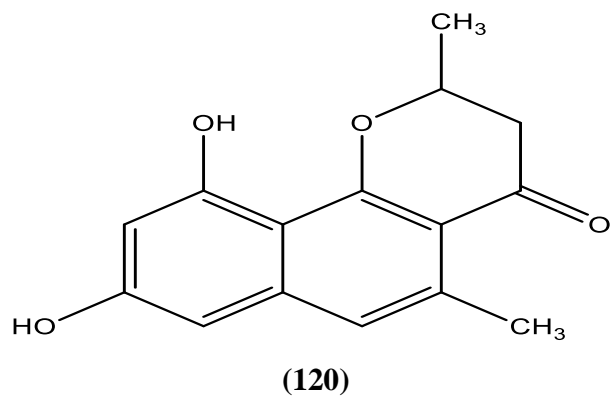

Fig. 2.5. Structure of compound 120 
<smiles>CCCCCC(CC)COC(=O)c1ccccc1C(=O)OCC(CC)CCCC</smiles>

(121)

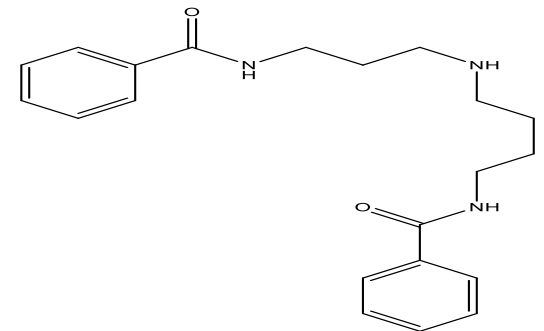

(122)

(123)<smiles>CCCCCCCCCCCCCCCC(=O)O</smiles>

(124)<smiles>CCCCCCCCCCCCCCCCCC(=O)O</smiles><smiles>CCCCCCCC/C=C/CCCCCCCC(=O)O</smiles><smiles>CCCCC/C=C\C/C=C\CCCCCCCC(=O)O</smiles>

(127)<smiles>CC/C=C/C/C=C/C/C=C/CCCCCCCC(=O)O</smiles>

(128)<smiles>CCCCCCCCCCCCCCCCCCCC(=O)O</smiles>

Fig. 3.1. Structure of compounds 121- 128 


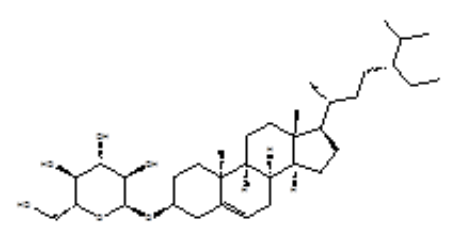

(129)

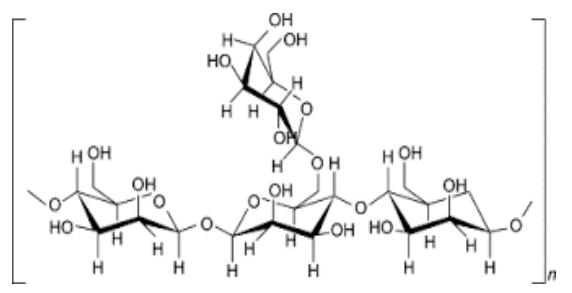

(130)

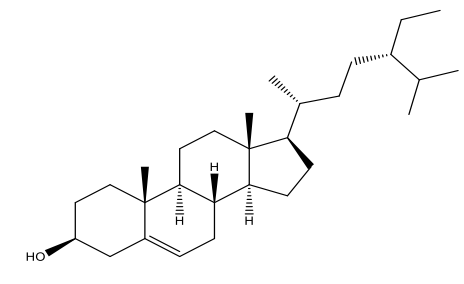

(131)

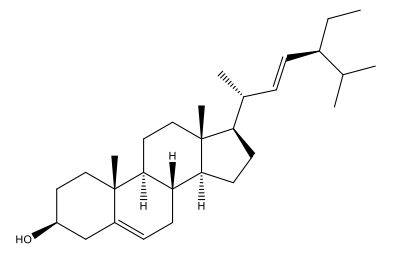

(132)

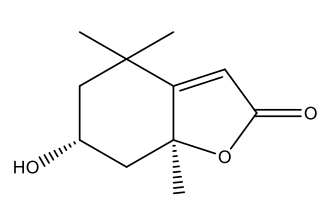

(133)

Fig. 3.2. Structure of compounds 129- 133<smiles>[R4]c1cc([R3])c([R2])c([R3])c1</smiles>

$\mathbf{R}_{1}$ $\mathbf{R}_{2}$

$\mathbf{R}_{3}$

$\mathbf{R}_{4}$

(134)

$\mathrm{H}$

$\mathrm{H}$

$\mathrm{H}$

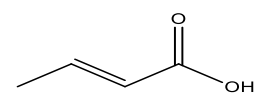

(135)

$\mathrm{OMe}$

$\mathrm{OH}$

$\mathrm{H}$

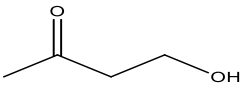

(136)

$\mathrm{OMe}$

$\mathrm{OH}$

$\mathrm{H}$

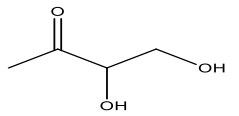

(137)

OMe

$\mathrm{OH}$

$\mathrm{OMe}$

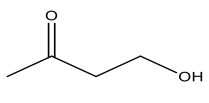

(138)

$\mathrm{OMe}$

$\mathrm{OH}$

$\mathrm{OMe}$

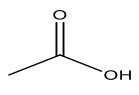

(139)

$\mathrm{OMe}$

$\mathrm{OH}$

$\mathrm{H}$

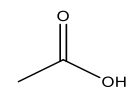

Fig. 3.3. Structure of compounds 134- 139 


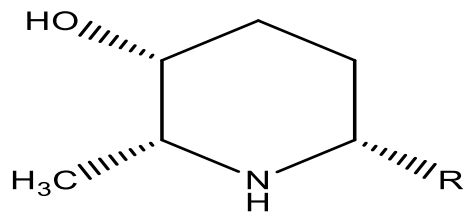

$\mathbf{R}$

(140)

(142)

$$
\begin{gathered}
-\left(\mathrm{CH}_{2}\right)_{12} \mathrm{COCH} 3 \\
-\left(\mathrm{CH}_{2}\right)_{12} \mathrm{CO}(\mathrm{OH}) \mathrm{CH}_{3} \\
-\left(\mathrm{CH}_{2}\right)_{10} \mathrm{CO}(\mathrm{OH}) \mathrm{CH}_{3}
\end{gathered}
$$

Fig. 3.4. Structure of compounds 140- 142

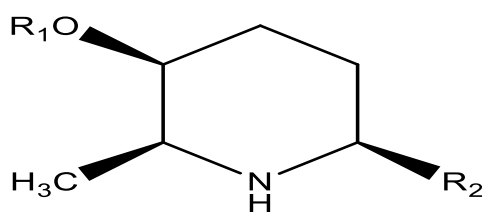

$\mathbf{R}_{1}$

$\mathrm{H}$

Ac

$\mathrm{H}$
$\mathbf{R}_{2}$

$-\left(\mathrm{CH}_{2}\right)_{10} \mathrm{CO}(\mathrm{OH}) \mathrm{CH}_{2} \mathrm{OH}$

$-\left(\mathrm{CH}_{2}\right)_{10} \mathrm{CO}(\mathrm{OH}) \mathrm{CH}_{2} \mathrm{OH}$

$-\left(\mathrm{CH}_{2}\right)_{10} \mathrm{COCH} 3$

Fig. 3.5. Structure of compounds143- 145

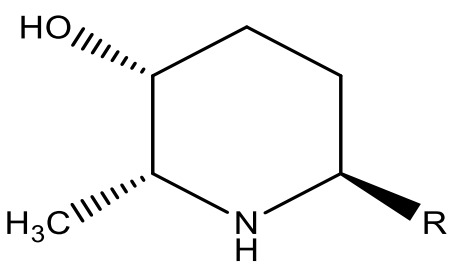

$\mathbf{R}$

(146)

$$
-\left(\mathrm{CH}_{2}\right)_{10} \mathrm{CO}(\mathrm{OH}) \mathrm{CH}_{3}
$$

(147)

$$
-\left(\mathrm{CH}_{2}\right)_{10} \mathrm{CO}_{2} \mathrm{H}
$$

Fig. 3.6. Structure of compounds 146 and 147 
<smiles>CC(=O)Cc1cc(O)cc2oc(C)cc(=O)c12</smiles>

(148)

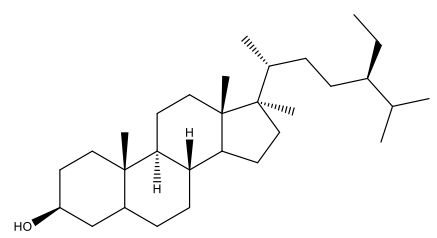

(151)
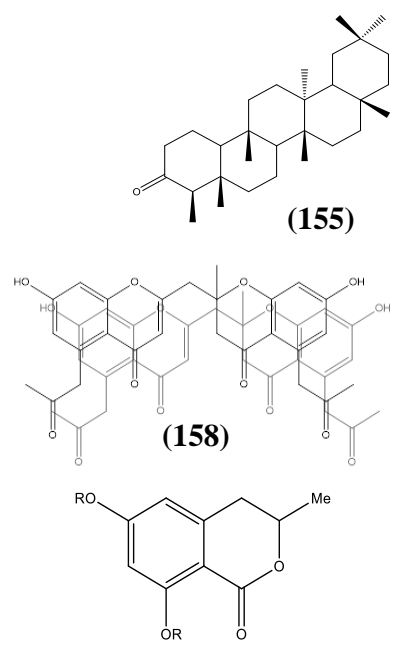

(161) $\mathrm{R}=\mathrm{H}$

(162) $\mathrm{R}=\mathrm{Ac}$

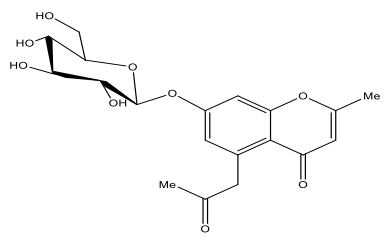

(149)

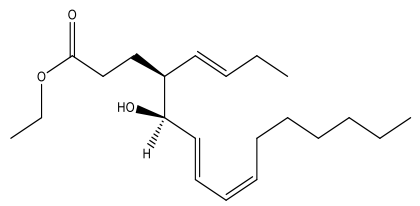

(152)

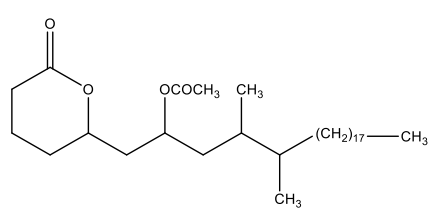

(150)

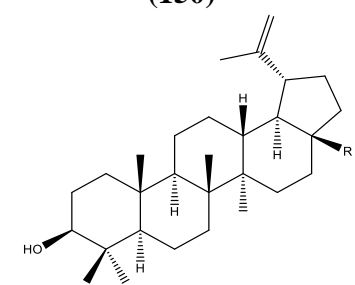

(153) $\mathrm{R}=\mathrm{CH}_{3}$ (154) $\mathrm{R}=\mathrm{COOH}$

Fig. 3.7. Structure of compounds 148 - 154
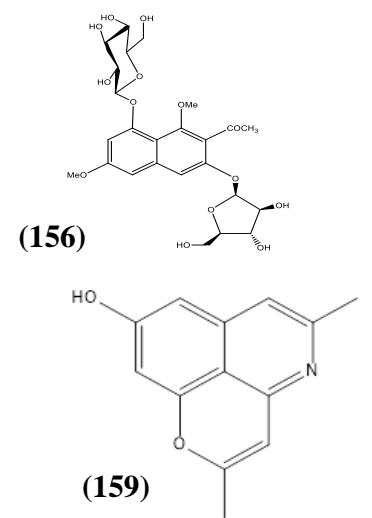

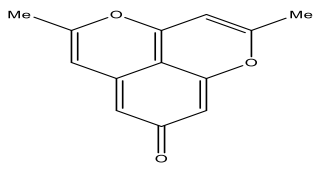

(163)

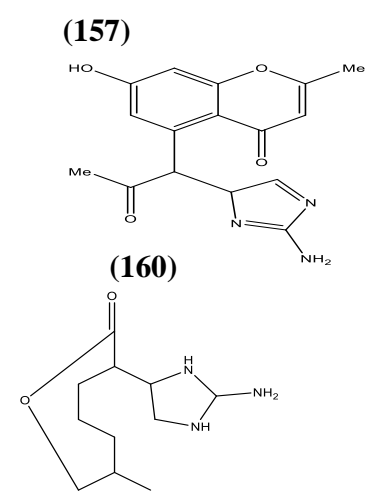

Fig. 3.8. Structure of compounds 155- 163

Fig. 3. The chemical structure of reported some miscellaneous compounds have been isolated from different Cassia species in Fig. 3 (Fig.3.1 - Fig.3.8). 


\section{Declarations}

\section{Ethics approval and consent to participate}

Not applicable

\section{Consent to publish}

Not applicable

\section{Consent to publish}

Not applicable

\section{Availability of data and materials}

All data generated or analyzed during this study are included in this published article in the main manuscript.

\section{Competing interests}

The authors declare that no competing interests exist

\section{Funding Statement}

No funding source was received.

\section{Authors' contributions}

The manuscript was drafted and written by all authors. All authors have read and approved the final manuscript.

\section{Acknowledgment}

The authors would like to acknowledge all colleagues in the Department of Pharmacognosy, Faculty of Pharmacy, Ain-Shams University for their constructive comments.

\section{REFERENCES}

1. Tene, V., Malagon, O., Finzi, P. V., Vidari, G., Armijos, C., and Zaragoza, T. An ethnobotanical survey of medicinal plants used in Loja and Zamora-Chinchipe, Ecuador. J. Ethnopharmacol. 2007; 111(1), 63-81.

\section{https://doi.org/10.1016/j.jep.2006.10.032}

2. Hu, J.-M., Lavin, M., Wojciechowski, M. F., and Sanderson, M. J. Phylogenetic systematics of the tribe Millettieae (Leguminosae) based on chloroplast trnK/matK sequences and its implications for evolutionary patterns in
Papilionoideae. Am. J. Bot. 2000; 87(3), 418-430. https://doi.org/10.2307/2656638

3. Awomukwu, D.A., Nyananyo, B.L., Ikpeama, A.I. and Adieze, C.U., Comparative chemical constituents of some Cassia species and their pharmacognostic importance in South-Eastern Nigeria. Sci. J. of Chemi. 2015; 3(3), pp.40-49.

4. Gledhill, D. (2008). The names of plants. Cambridge University Press.

5. Willis C., (1973). A dictionary of the flowering plants and frens. 8th ed., Cambridge University Press. The USA.

6. Dave, H., and Ledwani, L. A review on anthraquinones isolated from Cassia species and their applications. Ind. J. Nat. Prod. Resour. 2012; Vol. 3(3), pp. 291-319.

\section{http://hdl.handle.net/123456789/14810}

7. Caro, Y., Anamale, L., Fouillaud, M., Laurent, P., Petit, T. and Dufosse, L., Natural hydroxyanthraquinoid pigments as potent foodgrade colorants: an overview. Natural Products and Bioprospecting. J. Nat. Prod. 2012; 2(5), pp.174193.

https://doi.org/10.1016/j.copbio.2013.09.007

8. Kalidhar, S. B., and others. Alatinone, an anthraquinone from Cassia alata. Phytochem.1993; 32(6), 1616-1617.

https://doi.org/10.1016/0031-9422(93)85192-T

9. Fernand, V. E., Dinh, D. T., Washington, S. J., Fakayode, S. O., Losso, J. N., van Ravenswaay, R. O., and Warner, I. M. Determination of pharmacologically active compounds in root extracts of Cassia alata L. by use of highperformance liquid chromatography. Talanta, 2008; 74(4), 896-902.

\section{https://doi.org/10.1016/j.talanta.2007.07.033}

10. Verma, R. K., Uniyal, G. C., Singh, S. P., Sharma, J. R., and Gupta, M. M. Reverse-phase HighPerformance Liquid Chromatography of Sennosides in Cassia angustifolia. Phytoch. Anal. 1996; 7(2), 73-75.

\section{https://doi.org/10.1080/14786419.2016.1258562}

11. Kinjo, J., Ikeda, T., Watanabe, K., and Nohara, T. An anthraquinone glycoside from Cassia angustifolia leaves. Phytochem. 1994; 37(6), 1685-1687. 
https://doi.org/10.1016/S0031-9422(00)89592-8

12. Barba, B., D।'liaz, J. G., \& Herz, W. Anthraquinones and other constituents of two Senna species. Phytochem. 1992; 31(12), 43744375.

https://doi.org/10.1016/0031-9422(92)80483-U

13. Alemayehu, G., Hailu, A., and Abegaz, B. M. Bianthraquinones from Senna didymobotrya. Phytochem. 1996a; 42(5), 1423-1425.

https://doi.org/10.1016/0031-9422(96)00102-1

14. Tiwari, H.P., and Misra, M., 1993. Phytochemical investigation of Cassia glauca bark. J. Ind. Chem. Soc. 1993; 70(7), pp.653-653.

15. Kalidhar, S. B., and others. Chemical components of Cassia glauca lam. Ind. J. of Pharma. Sci.1994; $56(1)$.

16. Gonza, A. G., Barrera, J. B., Davila, B. B., Valencia, E., Domi, X. A., \& others. Anthraquinones from Cassia greggii. Phytochem.1992; 31(1), 255-258.

https://doi.org/10.1016/0031-9422(91)83048-P

17. Anu, S. J., \& Rao, J. M. Oxanthrone esters from the roots of Cassia kleinii. Phytochem. 2002; 59(4), 425-427.

https://doi.org/10.1016/S0031-9422(01)00439-3

18. Alemayehu, G., Abegaz, B., Snatzke, G., and Duddeck, H. Bianthrones from Senna longiracemosa. Phytochem. 1993; 32(5), 12731277.

https://doi.org/10.1016/S0031-9422(00)95104-5

19. Alemayehu, G., and Abegaz, B. M. Bianthraquinones from the seeds of Senna multiglandulosa. Phytochem. 1996b; 41(3), 919921.

https://doi.org/10.1016/0031-9422(95)00645-1

20. Abegaz, B. M., Bezabeh, M., Alemayehu, G., \& Duddeck, H. Anthraquinones from Senna multiglandulosa. Phytochem. 1994; 35(2), 465468.

https://doi.org/10.1016/S0031-9422(00)94783-6

21. Yadav, J.P., Arya, V., Yadav, S., Panghal, M., Kumar, S. and Dhankhar, S., Cassia occidentalis L.: A review on its ethnobotany, phytochemical and pharmacological profile. Fitoterapia, 2010;
81(4), pp.223-230.

https://doi.org/10.1016/j.fitote.2009.09.008

22. Sob, S. V. T., Wabo, H. K., Tchinda, A. T., Tane, P., Ngadjui, B. T., Ye, Y., \& others. (2010). Anthraquinones, sterols, triterpenoids, and xanthones from Cassia obtusifolia. Biochem. Syst. Ecol. 2010; 38(3), 342-345.

http://dx.doi.org/10.1016/j.bse.2010.02.002

23. Sob, S. V. T., Wabo, H. K., Tane, P., Ngadjui, B. $\mathrm{T}$., and $\mathrm{Ma}, \mathrm{D}$. A xanthone and a polyketide derivative from the leaves of Cassia obtusifolia (Leguminosae). Tetrahedron, 2008; 64(34), 7999_ 8002 .

https://doi.org/10.1016/j.tet.2008.05.125

24. Sekar, M., Prasad, K. J. R., Sidduraju, P., and Janardhanan, K. New anthraquinones from Cassia obtusa. Fitoterapia, 1999; 70(3), 330-332.

https://doi.org/10.1016/S0367-326X(99)00041-6

25. Messana, I., Ferrari, F., Cavalcanti, M. S. B., and Morace, G. An anthraquinone and three naphthopyrone derivatives from Cassia pudibunda. Phytochem. 1991; 30(2), 708-710.

https://doi.org/10.1016/0031-9422(91)83762-A

26. El-Toumy, S. A., El Souda, S. S., Mohamed, T. K., Brouard, I., and Bermejo, J. Anthraquinone glycosides from Cassia roxburghii and evaluation of its free radical scavenging activity. Carbohydr. Res. 2012; 360, 47-51.

https://doi.org/10.1016/j.carres.2012.07.020

27. Nair, M. S. R., McMorris, T. C., and Anchel, M. Cassiaxanthone, a hydroxyxanthone dicarboxylic acid from Cassia species. Phytochem. 1970; 9(5), 1153-1155.

https://doi.org/10.1016/S0031-9422(00)85246-2

28. Singh, V., Singh, J., and Sharma, J. P. Anthraquinones from heartwood of Cassia siamea. Phytochem. 1992; 31(6), 2176-2177.

https://doi.org/10.1016/0031-9422(92)80395-U

29. Koyama, J., Morita, I., Tagahara, K., and Aqil, M. Bianthraquinones from Cassia siamea. Phytochem. 2001; 56(8), 849-851.

https://doi.org/10.1016/S0031-9422(01)00025-5

30. Sui-Ming, W., Wong, M. M., Seligmann, O., and Wagner, H. Anthraquinone glycosides from the seeds of Cassia tora. Phytochem. 1989; 28(1), 
211-214.

https://doi.org/10.1016/0031-9422(89)85040-X

31.Zhu, L., Yu, S., Zeng, X., Fu, X., \& Zhao, M. Preparative separation and purification of five anthraquinones from Cassia tora L. by high-speed counter-current chromatography. Sep. Purif. Tech. 2008; 63(3), 665-669.

https://doi.org/10.1016/j.seppur.2008.07.011

32. Kitanaka, S., \& Takido, M. (S)-5, 7'-biphyscion 8glucoside from Cassia torosa. Phytochem. 1995; 39(3), 717-718.

\section{https://doi.org/10.1016/0031-9422(94)00955-S}

33. Takahashi, S., Kitanaka, S., Takido, M., Sankawa, U., and Shibata, S. Phlegmacins and anhydrophlegmacinquinones: Dimeric hydroanthracenes from seedlings of Cassia torosa. Phytochem. (UK) 1977; 16(7), 999-1002.

\section{https://doi.org/10.1016/S0031-9422(00)86709-6}

34. Takido, M., Kitanaka, S., Takahashi, S., and Tanaka, T. Germitorosone and methylgermitorosone, two hydroanthracene derivatives from seedlings of Cassia torosa. Phytochem.1982; 21(2), 425-427.

\section{https://doi.org/10.1016/S0031-9422(00)95281-6}

35. Gupta, D., and Singh, J. Flavonoid glycosides from Cassia alata. Phytochem.1991; 30(8), 2761-2763.

https://doi.org/10.1016/0031-9422(91)85140-U

36. Juan-Badaturuge, M., Habtemariam, S., and Thomas, M. J. K. Antioxidant compounds from a South Asian beverage and medicinal plant, Cassia auriculata. Food Chem. 2011; 125(1), 221-225.

https://doi.org/10.1016/j.foodchem.2010.08.065

37. Ganapaty, S., Thomas, P.S., Ramana, K.V., Vidyadhar, K.N. and Chakradhar, V., A review of phytochemical studies of Cassia species. J. of Nat. Remedies. 2002; 2(2), pp.102-120.

38. Nagpal, M. A., Nagpal, N., Rahar, S., Shah, G., Swami, G., Kapoor, R., and others. Phytochemical investigation of methanolic extract of Cassia fistula leaves. J. Pharmacog. 2011; 3(26), 61-69.

https://doi.org/10.5530/pj.2011.26.11

39. El-Sayed, M. M., Abdel-Aziz, M. M., AbdelGawad, M. M., Abdel-Hameed, E. S., Ahmed, W. S., and Abdel-Lateef, E. E. Chemical constituents and cytotoxic activity of Cassia glauca Lan.
Leaves. Life. Sci. J. 2013; 10(3), 1617-1625.

40.El-Sawi, S. A., Sleem, A. A., and others. Flavonoids and hepatoprotective activity of leaves of Senna surattensis (Burm. f.) in CCl4 induced hepatotoxicity in rats. Aust. J. Basic Appl. Sci. 2010; 4(6), 1326-1333.

http://www.insipub.com/ajbas/2010/1326-1333.pdf

41. Murakami, S., Arai, I., Muramatsu, M., Otomo, S., Baba, K., Kido, T., and Kozawa, M. Inhibition of gastric $\mathrm{H}+, \mathrm{K}+$-ATPase and acid secretion by cassigarol A, a polyphenol from Cassia garrettiana Craib. Biochem. Pharmacol. 1992; 44(1), 33-37.

https://doi.org/10.1016/0006-2952(92)90034-G

42. Baba, K., Kido, T., Maeda, K., Taniguchi, M., \& Kozawa, M. Two stilbenoids from Cassia garrettiana. Phytochem.1992; 31(9), 3215-3218.

https://doi.org/10.1016/0031-9422(92)83478-H

43. Baba, K., Kido, T., Taniguchi, M., and Kozawaqa, M. Stilbenoids from Cassia garrettiana. Phytochem.1994; 36(6), 1509-1513.

https://doi.org/10.1016/S0031-9422(00)89752-6

44. Mohamed, G. A. New cytotoxic cycloartane triterpene from Cassia italica aerial parts. Nat. Prod. Res. 2014; 28(13), 976-983.

https://doi.org/10.1080/14786419.2014.902820

45. Sharma, A., Ahmad, S. and Harikumar, S.L., Pharmacognosy, Phytochemistry and Pharmacology of Cassia javanica Linn: A Review. J. Pharma Res. Rev. 2014; 3(4), pp.101-105.

Aditi Sharma et.al, IJPRR 2014; 3(4)

46. Kitanaka, S., and Takido, M. Demethyltorosaflavones C and D from Cassia nomame. Phytochem. 1992; 31(8), 2927-2929.

https://doi.org/10.1016/0031-9422(92)83671-K

47. Wu, X., Ruan, J., Yang, V. C., Wu, Z., Lou, J., Duan, H., Guo, D. Three new acetylated benzyl- $\beta$ -resorcylate glycosides from Cassia obtusifolia. Fitoterapia. 2012; 83(1), 166-169.

https://doi.org/10.1016/j.fitote.2011.10.009

48. Rao, G. N., Kumar, P. M., Dhandapani, V. S., Krishna, T. R., and Hayashi, T. Constituents of Cassia auriculata. Fitoterapia. 2000; 71(1), 8283.

https://doi.org/10.1016/S0367-326X(99)00108-2 
49. Singh, S., Singh, S.K. and Yadav, A., A review on Cassia species: Pharmacological, traditional and medicinal aspects in various countries. $J$. American. Phytomed. Clinical Therap. 2013; 1(3), pp.291-312.

50. Dixit, a. k., and tiwari, h. p. Fatty acid composition and characteristics of Cassia glauca seed oil. J. Indian Chem. Soc. 1990; 67(10).

51. Kazmi, M. H., Malik, A., Hameed, S., Akhtar, N., and Ali, S. N. An anthraquinone derivative from Cassia italica. Phytochem. 1994; 36(3), 761-763.

https://doi.org/10.1016/S0031-9422(00)89812-X

52. Jones, L., Bartholomew, B., Latif, Z., Sarker, S. D., \& Nash, R. J. Constituents of Cassia laevigata. Fitoterapia. 2000; 71(5), 580-583.

https://doi.org/10.1016/S0367-326X(00)00155-6

53. Bolzani, V. da S., Gunatilaka, A. A. L., and Kingston, D. G. I. Bioactive and other piperidine alkaloids from Cassia leptophylla. Tetrahedron. 1995; 51(21), 5929-5934.

\section{https://doi.org/10.1016/0040-4020(95)00254-6}

54. Zhao, Y., Zhao, K., Jiang, K., Tao, S., Li, Y., Chen, W., Kou, S., Gu, C., Li, Z., Guo, L. and L White, W., A review of flavonoids from Cassia species and their biological activity. Current Pharm. Biotechno. 2016; 17(13), pp.1134-1146.

https://doi.org/10.2174/1389201017666160819151153

55. Jain, R., Agrawal, O.P., Jain, N.K. and Jain, S.C., Phytochemical and biological evaluation of Cassia nodosa root bark. J. Indian Chem. Soc. 2013; 90(12), pp.2287-2290.

56. Rahman, M.M., Sultana, T., Ali, M.Y., Rahman, M.M., Al-Reza, S.M. and Rahman, A., Chemical composition and antibacterial activity of the essential oil and various extracts from Cassia sophera L. against Bacillus sp. from soil. Arabian J. Chem. 2017; 10, pp.S2132-S2137.

https://doi.org/10.1016/j.arabjc.2013.07.045

57. Choi, J. S., Jung, J. H., Lee, H. J., Lee, J. H., and Kang, S. S. A naphthalene glycoside from Cassia tora. Phytochem. 1995; 40(3), 997-999.

https://doi.org/10.1016/0031-9422(95)00318-2

58. Oshimi, S., Tomizawa, Y., Hirasawa, Y., Honda, T., Ekasari, W., Widyawaruyanti, A.Morita, H. Chrobisiamone A, a new bischromone from Cassia siamea and a biomimetic transformation of 5acetonyl-7-hydroxy-2-methylchromone into cassiarin A. Bioorg. Med. Chem. Lett. 2008; 18(13), 3761-3763.

https://doi.org/10.1016/j.bmcl.2008.05.041

59. Ravi, K.J., Ganga, R.B., Lakshmi, N.M. and Mallikarjun, R.T., Evaluation of antidiabetic activity of Cassia siamea leaves in alloxan induced diabetic rats. J. Phytopharm. 2013; 4, pp.237-40.

60. Thongsaard, W., Deachapunya, C., Pongsakorn, S., Boyd, E. A., Bennett, G. W., and Marsden, C. A. Barakol: a potential anxiolytic extracted from Cassia siamea. Pharmacol. Biochem. Behav. 1996; 53(3), 753-758.

https://doi.org/10.1016/0091-3057(95)02088-8

61. Sobeh, M., Mahmoud, M.F., Abdelfattah, M.A., Cheng, H., El-Shazly, A.M. and Wink, M., A proanthocyanidin-rich extract from Cassia abbreviata exhibits antioxidant and hepatoprotective activities in vivo. $J$. Ethnopharmacol. 2018; 213(1), pp.38-47.

https://doi.org/10.1016/j.jep.2017.11.007

62. Kumaran, A., and Karunakaran, R. J. Antioxidant activity of Cassia auriculata flowers. Fitoterapia. 2007; 78(1), 46- 47.

https://doi.org/10.1016/j.fitote.2006.09.031

63. Deshpande, S., Kewatkar, S. M., and Paithankar, V. V. In-vitro antioxidant activity of different fraction of roots of Cassia auriculata Linn. Drug Inven. Tod. 2013; 5(2), 164-168.

https://doi.org/10.1016/j.dit.2013.05.006

64. Bharathi, D. and Bhuvaneshwari, V., Evaluation of the Cytotoxic and Antioxidant Activity of Phytosynthesized Silver Nanoparticles Using Cassia angustifolia Flowers. J. BionanoSci. 2019; 9(1), pp.155-163.

https://doi.org/10.1007/s12668-018-0577-5

65. Siddhuraju, P., Mohan, P. S., and Becker, K. Studies on the antioxidant activity of Indian Laburnum (Cassia fistula L.): a preliminary assessment of crude extracts from stem bark, leaves, flowers and fruit pulp. Food Chem. 2002; 79(1), 61-67.

https://doi.org/10.1016/S0308-8146(02)00179-6

66. Limtrakul, P., Yodkeeree, S., Thippraphan, P., Punfa, W. and Srisomboon, J., Anti-aging and tyrosinase inhibition effects of Cassia fistula flower butanolic extract. BMC Complem. Altern. med. 2016; 16(1), p.497. 
https://doi.org/10.1186/s12906-016-1484-3

67. Srividhya, M., Hridya, H., Shanthi, V. and Ramanathan, K., Bioactive Amento flavone isolated from Cassia fistula L. Leaves exhibits therapeutic efficacy. 3 Biotech. 2017; 7(1), p.33.

http://creativecommons.org/licenses/by/4.0/

68. Sharma, D.K., Enumerations on phytochemical, pharmacological and ethnobotanical properties of Cassia fistula Linn: yellow shower. Seeds. 2017; 6(7), p.8.

69. El-hashash, M., Abdel-Gawad, M., El-Sayed, M., Sabry, W., Abdel-Hameed, E.-S., and AbdelLateef, E. Antioxidant properties of methanolic extracts of the leaves of seven Egyptian Cassia species. Acta Pharmaceutica. 2010; 60(3), 361367.

https://doi.org/10.2478/v10007-010-0030-y

70. Afify, A. E.-M. M. R., and Hassan, H. M. M. Free radical scavenging activity of three different flowers-Hibiscus rosa-sinensis, Quisqualis indica and Senna surattensis. Asi. Pacif. J. Trop. Biomed. 2016; 6(9), 771-777.

https://doi.org/10.1016/j.apjtb.2016.07.006

71. Veerapur, V. P., Pratap, V., Thippeswamy, B. S., Marietta, P., Bansal, P., Kulkarni, P. V, and Kulkarni, V. H. Polyphenolic enriched extract of Cassia glauca Lamk, improves streptozotocininduced type-1 diabetes linked with partial insulin resistance in rats. J. Ethnopharmacol. 2017; 198(23), 489-498.

https://doi.org/10.1016/j.jep.2017.01.025

72. Kumar, D., Singh, A., Sanghi, A., Chandra, R., and Arora, S. Individual and combined effects of leaves and flowers extracts of Cassia glauca on membrane stabilization, antimicrobial and antioxidant activities. J. Pharma. Sci. 2017b; 8(2), 129-134.

https://www.researchgate.net/journal/09757538_International_Journal_of_Research_in_Phar maceutical_Sciences

73. Sangetha, S., Sasidharan, S., Zuraini, Z., and Suryani, S. Antioxidant activity of methanolic extracts of Cassia surattensis. Pharmacol. online. 2008b; 2, 829-838.

74. Kumar, U., Seeta, U., Chen, Y., Kanwar, J. R., and Sasidharan, S. Redox Control of Antioxidant and Antihepatotoxic Activities of Cassia surattensis
Seed Extract against Paracetamol Intoxication in Mice: In Vitro and In Vivo Studies of Herbal Green Antioxidant. Oxid. Med. Cell. Longevity. 2016; 2016. 13 pages.

http://dx.doi.org/10.1155/2016/6841348

75. Chew, Y.-L., Goh, J.-K., and Lim, Y.-Y. Assessment of in vitro antioxidant capacity and polyphenolic composition of selected medicinal herbs from Leguminosae family in Peninsular Malaysia. Food Chem. 2009; 116(1), 13-18.

https://doi.org/10.1016/j.foodchem.2009.01.091

76. Sundaramoorthy, S.,Gunasekaran, S., Arunachalam, S. and Sathiavelu, M., A phytopharmacological review on Cassia species. $J$. Pharma. Sci. and Res. 2016; 8(5), p.260.

77. Mehta, J.P., Parmar, P.H., Vadia, S.H., Patel, M.K. and Tripathi, C.B., In-vitro antioxidant and in-vivo anti-inflammatory activities of aerial parts of Cassia species. Arabian J. Chem. 2017; 10(2), pp.S1654-S1662.

https://doi.org/10.1016/j.arabjc.2013.06.010

78. Kaur, G., Alam, M. S., Jabbar, Z., Javed, K., and Athar, M. Evaluation of antioxidant activity of Cassia siamea flowers. J. Ethnopharmacol. 2006; 108(3), 340-348.

https://doi.org/10.1016/j.jep.2006.05.021

79. Dong, X., Fu, J., Yin, X., Yang, C., Zhang, X., Wang, W., Du, X., Wang, Q. and Ni, J., Cassiae semen: A review of its phytochemistry and pharmacology. Mol. Med. Rep. 2017; 16(3), pp.2331-2346.

https://doi.org/10.3892/mmr.2017.6880

80. Zhenbao, J., Fei, T., Ling, G., Guanjun, T., and Xiaolin, D. Antioxidant properties of extracts from juemingzi (Cassia tora L.) evaluated in vitro. LWT-Food Sci. Tech. 2007; 40(6), 1072-1077.

https://doi.org/10.1016/j.lwt.2006.05.010

81. Chethana, K.R., Senol, F.S., Orhan, I.E., Anilakumar, K.R. and Keri, R.S., Cassia tora Linn: A boon to Alzheimer's disease for its antiamyloidogenic and cholinergic activities. Phytomed. 2017; 33(15), pp.43-52.

https://doi.org/10.1016/j.phymed.2017.06.002

82. Palanichamy, S., Nagarajan, S., and Devasagayam, M. Effect of Cassia alata leaf extract on hyperglycemic rats. J. Ethnopharmacol. 1988; 
22(1), 81-90.

https://doi.org/10.1016/0378-8741(88)90233-4

83. Gupta, S., Sharma, S. B., Bansal, S. K., and Prabhu, K. M. Antihyperglycemic and hypolipidemic activity of aqueous extract of Cassia auriculata L. leaves in experimental diabetes. J. Ethnopharmacol. 2009; 123(3), 499503.

\section{https://doi.org/10.1016/j.jep.2009.02.019}

84. Fauzi, F.M., John, C.M., Karunanidhi, A., Mussa, H.Y., Ramasamy, R., Adam, A. and Bender, A., Understanding the mode-of-action of Cassia auriculata via in silico and in vivo studies towards validating it as a long term therapy for type II diabetes. J. Ethnopharmacol. 2017; 197(2), pp.6172.

https://doi.org/10.1016/j.jep.2016.07.058

85. Farswan, M., Mazumder, P. M., and Percha, V. Protective effect of Cassia glauca Linn. On the serum glucose and hepatic enzymes level in streptozotocin induced NIDDM in rats. J. Ind. Pharmacol. 2009; 41(1), 19.

\section{https://doi.org/10.4103/0253-7613.48887}

86. Salahuddin, M., and Jalalpure, S. S. Evaluation of antidiabetic activity of Cassia glauca Lam. leaf in streptozotocin induced diabetic rats. Iran. J. Pharmacol. Therap. 2010b; 9(1), 20-29.

http://ijpt.iums.ac.ir/article-1-197-en.html

87. Salahuddin, M. D., Jalalpure, S. S., and Gadge, N. B. Antidiabetic activity of aqueous bark extract of Cassia glauca in streptozotocin-induced diabetic rats. Can. J. Physiol. Pharmacol. 2010a; 88(2), 153-160.

https://doi.org/10.1139/Y09-121

88. Petchi, R. R. Evaluation of anti-diabetic activity of Cassia surattensis burm. F. Flower in streptozotocin induced diabetic rats. J. Res. Pharma. Sci. 2016; 2(2), 200-205.

89. Thilagam, E., Parimaladevi, B., Kumarappan, C., and Mandal, S. C. $\$ \alpha \$-G l u c o s i d a s e$ and $\$ \alpha \$-$ amylase inhibitory activity of Senna surattensis. $J$. Acupun. Merid. Stud. 2013; 6(1), 24-30.

https://doi.org/10.1016/j.jams.2012.10.005

90. Kumar, D., Jain, A.,and Verma, A. Phytochemical and Pharmacological Investigation of Cassia Siamea Lamk: An Insight. J. Nat. Prod. 2017a; 7(4), 255-266. https://doi.org/10.2174/2210315507666170509125800

91. Adikay, S., and Santhoshini, T. Pharmacological evaluation of anti-ulcer activity of Cymbopogon flexuosus. J. Basic and Clin. Pharmacol. 2017; 4(2), 208-212.

92. Palanichamy, S., Nagarajan, S., and others. Antifungal activity of Cassia alata leaf extract. $J$. Ethnopharmacol. 1990b; 29(3), 337-340.

https://doi.org/10.1016/0378-8741(90)90043-s

93. Ntandou, G. F. N., Banzouzi, J. T., Mbatchi, B., Elion-Itou, R. D. G., Etou-Ossibi, A. W., Ramos, S., ... Ouamba, J. M. Analgesic and antiinflammatory effects of Cassia siamea Lam. stem bark extracts. J. Ethnopharmacol. 2010; 127(1), 108-111.

https://doi.org/10.1016/j.jep.2009.09.040

94. Chaudhari, S. S., Chaudhari, S. R., \& Chavan, M. J. Analgesic, anti-inflammatory and anti-arthritic activity of Cassia uniflora Mill. Asian Pac. J. Trop. Biomed. 2012; 2(1), S181--S186.

95. Cunha, L.C., de Morais, S.A., de Aquino, F.J., Chang, R., de Oliveira, A., Martins, M.M., Martins, C.H., Sousa, L.C., Barros, T.T., da Silva, C.V. and do Nascimento, E.A., Bioassay-guided fractionation and antimicrobial and cytotoxic activities of Cassia bakeriana extracts. Revista Brasileira de Farmacognosia. 2017; 27(1), pp.9198.

https://doi.org/10.3390/molecules18044588

96. Deepak, K., Shefali, A., and Ankit, V. Fatty acid composition and antimicrobial and antioxidant activity of Cassia glauca seed extracts. $J$. Phytopharmacol. 2013; 4(2), 113-118.

97. Voon, H. C., Bhat, R., and Rusul, G. Flower extracts and their essential oils as potential antimicrobial agents for food uses and pharmaceutical applications. Compr. Rev. Food Sci. Food Saf. 2012; 11(1), 34-55.

https://doi.org/10.1002/ptr.6101

98. Chukwujekwu, J. C., Coombes, P. H., Mulholland, D. A., and Van Staden, J. Emodin, an antibacterial anthraquinone from the roots of Cassia occidentalis. S.Afr. J. Bot. 2006; 72(2), 295-297.

https://doi.org/10.1016/j.sajb.2005.08.003

99. Palanichamy, S., Nagarajan, S., and others. Antifungal activity of Cassia alata leaf extract. $J$. Ethnopharmacol.1990b; 29(3), 337-340. 
https://doi.org/10.1016/0378-8741(90)90043-s

100. Sumathy, V., Zakaria, Z., Jothy, S. L., Gothai, S., Vijayarathna, S., Latha, L. Y., Sasidharan, S. In vitro and in vivo antifungal activity of Cassia surattensis flower against Aspergillus niger. Microb. Pathogen. 2014; 77, 712.

https://doi.org/10.1016/j.micpath.2014.10.004

101. Bhakta, T., Banerjee, S., Mandal, S. C., Maity, T. K., Saha, B. P., and Pal, M. Hepatoprotective activity of Cassia fistula leaf extract. Phytomed. 2001; 8(3), 220-224.

https://doi.org/10.1016/s0378-8741(98)00220-7

102. Ansari Asba, Bhot Meeta, Evaluation of Phytochemicals of Cassia tora Linn. And its cytotoxicity assay using Brine Shrimp. J. Pharmacog. Phytoch. Res. 2017; 9(4); 587-595.

DOI number: $10.25258 /$ phyto.v9i2.8132

103. Vijayaraj, P., Muthukumar, K., Sabarirajan, J., and Nachiappan, V. Antihyperlipidemic activity of Cassia auriculata flowers in triton WR 1339 induced hyperlipidemic rats. Exp. Toxicol. Patho. 2013; 65(1-2), 135-141.

https://doi.org/10.1016/j.etp.2011.07.001

104. Tzeng, T.-F., Lu, H.-J., Liou, S.-S., Chang, C. J., and Liu, I.-M. Reduction of lipid accumulation in white adipose tissues by Cassia tora (Leguminosae) seed extract is associated with AMPK activation. Food Chem. 2013; 136(2), 1086-1094.

https://doi.org/10.1016/j.foodchem.2012.09.017

105. Patil, U. K., Saraf, S., and Dixit, V. K. Hypolipidemic activity of seeds of Cassia tora Linn. J. Ethnopharmacol. 2004; 90(2-3), 249-252.

https://doi.org/10.1016/j.jep.2003.10.007

106. Aye, M.M., Aung, H.T., Sein, M.M. and Armijos, C., A Review on the Phytochemistry, Medicinal Properties and Pharmacological Activities of 15 Selected Myanmar Medicinal Plants. Molecules. 2019; 24(2), p.293.

https://doi.org/10.3390/molecules24020293

107. Bhakta, T., Banerjee, S., Mandal, S. C., Maity, T. K., Saha, B. P., and Pal, M. Hepatoprotective activity of Cassia fistula leaf extract. Phytomed. 2001; 8(3), 220-224. https://doi.org/10.1016/s0378-8741(98)00220-7

108. Maity, T. K., Mandal, S. C., Mukherjee, P. K., Saha, K., Das, J., Saha, B. P., and Pal, M. Evaluation of hepatoprotective potential of Cassia tora leaf extract. Nat. Prod. Sci. 1997; 3(2), 122126.

109. Jafri, M. A., Subhani, M. J., Javed, K., and Singh, S. Hepatoprotective activity of leaves of Cassia occidentalis against paracetamol and ethyl alcohol intoxication in rats. J. Ethnopharmacol. 1999; 66(3), 355-361.

https://doi.org/10.1016/s0378-8741(99)00037-9

110. John, C. M., Sandrasaigaran, P., Tong, C. K., Adam, A., and Ramasamy, R. Immunomodulatory activity of polyphenols derived from Cassia auriculata flowers in aged rats. Cell. Immunol. 2011; 271(2), 474-479.

https://doi.org/10.1016/j.cellimm.2011.08.017

111. Elujoba, A. A., Ajulo, O. O., and Iweibo, G. O. Chemical and biological analyses of Nigerian Cassia species for laxative activity. J. Pharma. Biomed. Anal. 1989; 7(12), 1453-1457.

https://doi.org/10.1016/0731-7085(89)80150-5 